\title{
De la crise humanitaire à la crise sécuritaire. Les dispositifs de contrôle des réfugiés syriens en Jordanie (2011-2015)
}

From a Humanitarian Crisis to a Security Crisis. Control Measures for Syrian Refugees in Jordan (2011-2015)

De la crisis humanitaria a la crisis de seguridad. Los dispositivos de vigilancia de los refugiados sirios en Jordania (2011-2015)

\section{Myriam Ababsa}

\section{(2) OpenEdition}

Journals

Édition électronique

URL : https://journals.openedition.org/remi/7380

DOI : $10.4000 /$ remi.7380

ISSN : $1777-5418$

Éditeur

Université de Poitiers

Édition imprimée

Date de publication : 1 décembre 2015

Pagination : 73-101

ISBN : 979-10-90426-26-9

ISSN : 0765-0752

Référence électronique

Myriam Ababsa, « De la crise humanitaire à la crise sécuritaire. Les dispositifs de contrôle des réfugiés syriens en Jordanie (2011-2015) », Revue européenne des migrations internationales [En ligne], vol. 31 n³ et 4 | 2015, mis en ligne le 01 décembre 2018, consulté le 14 avril 2022. URL : http:// journals.openedition.org/remi/7380 ; DOI : https://doi.org/10.4000/remi.7380 


\section{De la crise humanitaire à la crise sécuritaire. Les dispositifs de contrôle des réfugiés syriens en Jordanie (2011-2015)}

\section{Myriam Ababsa ${ }^{1}$}

Soutenue par les États-Unis, l'Europe et l'Arabie saoudite, la Jordanie est parvenue jusqu'en 2015 à contenir les récriminations d'une grande partie de la population face au déficit démocratique du jeu politique jordanien lors du printemps arabe, tout en jugulant une crise humanitaire considérable, née de l'afflux de plus d'1 million de réfugiés syriens. Mais, devant la réduction de l'assistance internationale aux réfugiés et aux Jordaniens les plus démunis et la pression que les réfugiés font peser sur les infrastructures scolaires et les services médicaux, la crise humanitaire se transforme en crise politique sur le plan intérieur et en crise sécuritaire. En effet, les 1,3 million de réfugiés syriens annoncés par le gouvernement jordanien - 629000 réfugiés enregistrés au Haut Commissariat aux Réfugiés (HCR) -, constituent près de $20 \%$ de l'ensemble de la population jordanienne ${ }^{2}$.

Membre temporaire du conseil de sécurité de I'ONU, la Jordanie a rejoint, en septembre 2014, les forces de la coalition qui combattent l'État islamique (EI) en Syrie et en Irak. La diffusion de la vidéo de I'assassinat du pilote jordanien Moath Al Kasasbeh par I'El en février 2015 a constitué un traumatisme national. Il s'est ensuivi un renforcement de l'appareil sécuritaire, la pendaison de deux djihadistes responsables des attentats de novembre 2005 et la réactivation de la loi antiterroriste de 2006. Les travaux de sécurisation des frontières conduits depuis 2014 ont été accélérés, avec l'installation de barrières et de miradors. Enfin, l'ordre a été donné à tous les réfugiés syriens présents dans le pays de se réenregistrer auprès des autorités. Selon des sources gouvernementales, environ 2000 Jordaniens combattraient en Syrie aux côtés de Jabhat al-Nusra ${ }^{3}$

\footnotetext{
1 Docteur en géographie, chercheure associée à I'Institut Français du Proche-Orient (IFPO), 3 I. Zahri Street, BP 830413-Zahran 11183, Amman, Jordanie ; m.ababsa@ ifporient.org

2 Selon le recensement national 2015, publié en février 2016, la population totale de la Jordanie est de 9,5 millions d'habitants, dont 1,265 million de réfugiés syriens. Cf. URL : http://www.jordantimes.com/news/local/population-stands-around-95-million-including29-million-guests

3 Jabhat al-Nusra (" le front de soutien ") est un groupe djihadiste syrien associé à Al-Qaida qui offre un soutien militaire aux rebelles de I'armée syrienne libre.
} 
et d'autres groupes affiliés à Al-Qaida et des milliers de salafistes - certains quiétistes, d'autres djihadistes - sont disséminés dans le pays.

Sous la pression de l'opinion publique, et parce qu'elle a décidé de s'engager militairement dans le combat contre I'EI, la Jordanie a durci sa politique envers les réfugiés syriens en 2014. L'interdiction de travail est maintenue, des contrôles sont conduits et les conditions de résidence sont de plus en plus restrictives. Cette pression croissante s'exerce sur les réfugiés syriens, alors que l'aide internationale se réduit et que les réfugiés ont épuisé leurs maigres ressources. L'assistance humanitaire islamique prend le relais avec une aide considérable fournie par les pays du Golfe. Le tiers des réfugiés est contraint de travailler dans des conditions illégales et bien souvent de déscolariser leurs enfants. La réduction de l'assistance alimentaire offerte par le Programme Alimentaire Mondial depuis l'été 2015 constitue un défi supplémentaire et nombreux sont les réfugiés qui n'ont d'autres solutions que de rejoindre les camps du HCR, de regagner la Syrie, ou pour certains de fuir vers l'Europe.

L'objectif de cet article est de présenter l'évolution de la politique de gestion jordanienne des réfugiés syriens depuis quatre ans, en mettant l'accent sur l'évolution des dispositifs de contrôle et des cadres légaux, sous la pression du contexte géopolitique. On montrera ainsi comment la présence des réfugiés conditionne désormais l'ensemble des politiques publiques, tant de protection sociale que de logement, ou de gouvernance locale. Ce travail illustrera la théorie géopolitique des mobilités forcées, selon laquelle les frontières sont plus poreuses aux flux de capitaux qu'aux hommes (Hyndman, 2000). Le travail s'appuie sur des entretiens conduits avec des officiels jordaniens et des réfugiés syriens entre février 2014 et février 2015 ainsi que sur des visites de camps ${ }^{4}$.

\section{D'une politique d'accueil généreuse au strict contrôle des migrants (2011-2014)}

Première terre d'accueil des réfugiés au monde, tant en chiffres absolus que relatifs avec 2 millions de réfugiés palestiniens en majorité citoyens et environ 400000 Irakiens dont 29300 réfugiés enregistrés au HCR ${ }^{5}$, la Jordanie a largement ouvert ses portes à ses voisins syriens dès mars 2011. Le régime a très tôt condamné Bachar Al Assad et appelé à son départ, montrant qu'il soutenait les aspirations démocratiques du peuple syrien, autant que celles des Jordaniens. Mais l'augmentation des réfugiés a conduit le gouvernement à

\footnotetext{
4 Deux rapports ont été préparés, I'un pour le HCR sur l'aide des donateurs du Golfe (UNHCR, 2015a), et I'autre pour le Norwegian Refugee Council sur le logement des réfugiés dans le Nord du pays (NRC, 2015).

5 Les réfugiés palestiniens bénéficient des services de I'UNRWA, I'agence des Nations unies en charge des réfugiés palestiniens, et sont aussi des citoyens jordaniens depuis 1949. En ajoutant les descendants non enregistrés et les Palestiniens originaires de Gaza arrivés en 1967, et qui ont des documents de voyage égyptiens, 45 \% de la population jordanienne est d'origine palestinienne (De Bel-Air, 2013). Le nombre de réfugiés irakiens est difficile à connaître, avec la multitude de retours vers I'Irak depuis 2013. Le gouvernement annonçait 750000 en 2005, mais une étude du centre de recherche norvégien Fafo (Forskningsstiftelsen Fafo) montrait que le pays comptait entre 450000 et 500000 Irakiens, dont plus de 300000 présents avant 2003, et moins de 50000 enregistrés au HCR (Fafo, 2007). En décembre 2015, ils sont 29300 enregiștrés par le HCR, une nouvelle vague arrivant de Bagdad et des territoires contrôlés par l'État islamique.
} 
contrôler plus étroitement les flux et les itinéraires, en créant des bureaux d'enregistrement puis des camps dès juillet 2012. Les réfugiés syriens en Jordanie enregistrés par le HCR étaient 628619 au 15 septembre $2015 ; 17 \%$ dans les trois camps du pays (Zaatari, Azraq et le camp émirien) et 519228 en milieu urbain. D'après la carte 1 , les camps ainsi que les réfugiés syriens vivant en milieu urbain (34 \% dans le gouvernorat d'Amman, $27 \%$ à Irbid, $14 \%$ à Mafraq, $9 \%$ à Zarqa) se trouvent essentiellement dans le Nord de la Jordanie, à proximité de la frontière syrienne ${ }^{6}$. Ceci confirme la règle suivant laquelle les réfugiés ont tendance à s'établir dans les pays voisins, dans les régions frontalières de leur pays d'origine. Selon les autorités jordaniennes, 700000 réfugiés syriens n'auraient pas souhaité s'enregistrer au HCR, soit un total de réfugiés (officiels et non enregistrés) oscillant selon les rapports officiels de 1,3 à 1,4 million.

Si le HCR les désigne du terme de réfugiés, ce n'est pas le cas du gouvernement jordanien qui les présente comme des "visiteurs ", des "invités ", des "invités irréguliers " ou des "frères arabes ". De nombreux pays arabes préfèrent garder le terme de réfugiés uniquement pour les Palestiniens qui ont perdu leur pays. Certains réfugiés syriens eux-mêmes refusent d'être appelés "réfugiés ", à la fois parce qu'ils sont arrivés comme immigrés et parce que leur pays reste à reconstruire. Afin que le mandat de I'UNRWA pour les réfugiés palestiniens garde tout son sens, la Jordanie n'est pas signataire de la convention de Genève de 1951 sur les réfugiés. En revanche, le statut des demandeurs d'asile est régi par un mémorandum avec le HCR datant de 1988, qui autorise cette agence à émettre des cartes de demandeurs d'asile. Selon la législation jordanienne, le statut de "réfugié " n'est accordé que si la personne obtient la réinstallation dans un pays tiers (qui n'est ni son pays d'origine, ni la Jordanie). II y a donc très peu de réfugiés au sens juridique du terme en Jordanie. Ce mémorandum a joué lors de la guerre en Irak et de l'arrivée de réfugiés irakiens dès 2003, puis en avril 2014. Il accepte le principe de non-refoulement, mais n'autorise pas l'installation permanente. L'article 21 de la constitution jordanienne offre l'asile politique à des catégories très restreintes de migrants forcés (Al Kilani, 2014) ${ }^{7}$.

\section{1 : I'arrivée de frontaliers activant des réseaux familiaux et de connaissance}

Quand la crise syrienne débuta en mars 2011, les premiers Syriens qui passèrent la frontière jordanienne, tracée en 1921 au milieu des plaines du Hauran, se rendirent provisoirement chez des proches, dans les gouvernorats d'Irbid et de Mafraq. Ils n'étaient alors pas considérés comme des réfugiés, la Jordanie autorisant les Syriens à entrer sur le territoire sans visa, à condition

6 UNHCR (2015) External Statistical Report on Active Registered Syrians, 15 September 2015, [online]. URL: http://data.unhcr.org/syrianrefugees/country.php?id=107

7 « The 1998 memorandum of understanding (MoU) between Jordan and UNHCR, amended in 2014, does not accept local integration as a solution for refugees and stipulates that "refugees" must be resettled within six months. People are legally considered " asylum seekers" and become "refugees" only after they have secured a resettlement space in a third country. Asylum seekers can be granted refugee status under article 21 of the Jordanian constitution, but only in very exceptional situations "(Carrion, $2015: 11$ ). 
Carte 1 : Distribution spatiale des réfugiés syriens enregistrés au HCR en septembre 2015

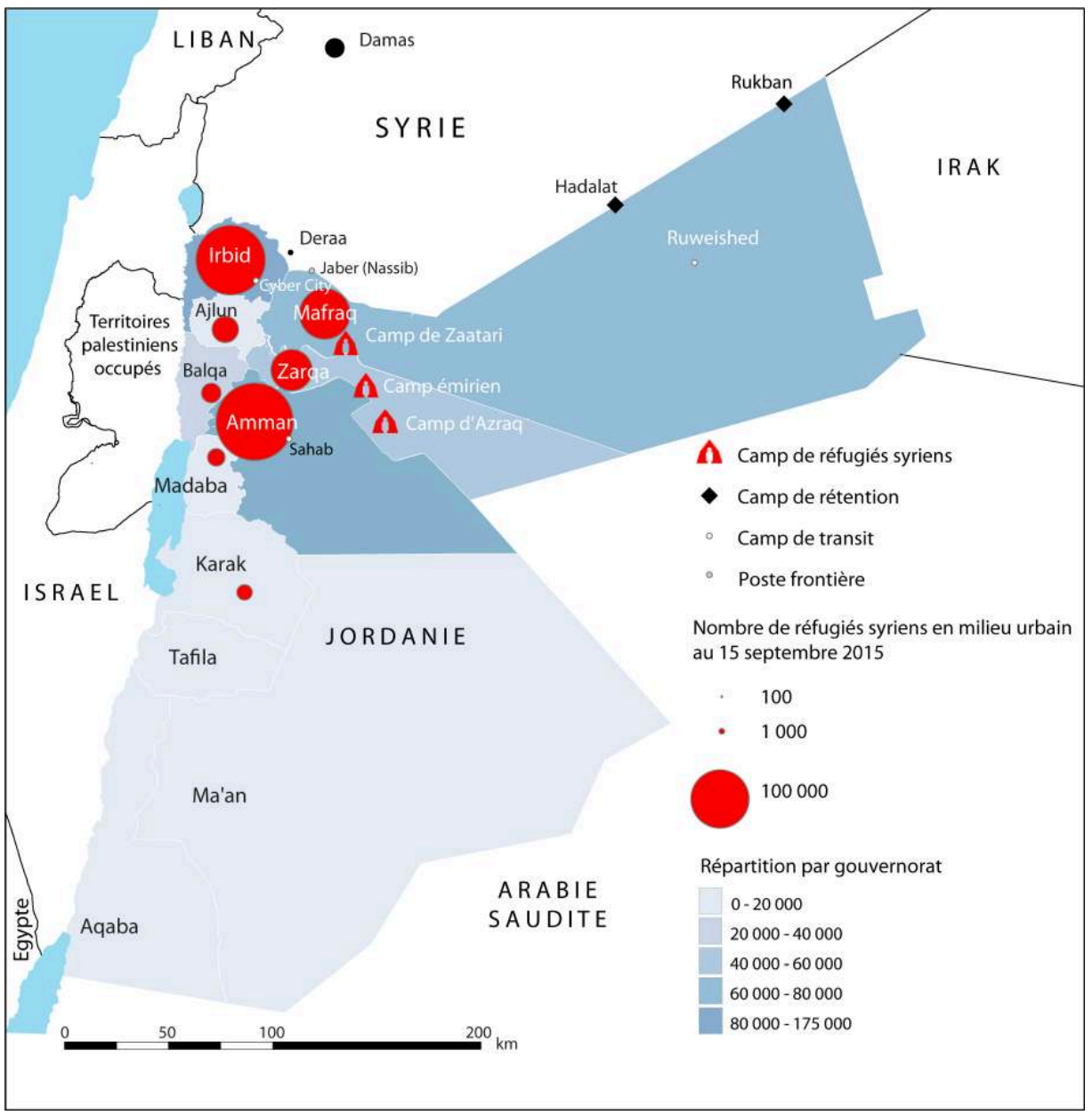

Source : UNHCR, External Statistical Report on Active Registered Syrians, 15 September 2015.

Crédit : M. Ababsa, 2015. 
qu'ils détiennent un passeport valide ${ }^{8}$. Sur les milliers qui partirent, seuls 2307 demandèrent I'asile (chiffres de décembre 2011) (UNHCR, 2014). En mars 2012, sur un panel de 105 Syriens, seuls $23 \%$ avaient fait une demande d'asile auprès du HCR (Olwan et Shiyab, 2012). Originaires du gouvernorat de Deraa, les familles de ces Syriens entretenaient des liens tribaux avec les Jordaniens du Nord $^{9}$. Les résultats de l'enquête montrent que si $30 \%$ des réfugiés justifient leur entrée en Jordanie pour la stabilité du pays, $20 \%$ par la proximité de la Syrie et la possibilité d'y retourner facilement, 16,6 \% disent y avoir de la famille (Olwan et Shiyab, 2012) ${ }^{10}$.

\section{2 : l'organisation des flux de réfugiés syriens par le gouvernement et le HCR}

Dès 2012, avec l'intensification des combats en Syrie, plus de 130000 Syriens arrivent en Jordanie. Pour un tiers d'entre eux, ce sont des raisons politiques qui justifient la migration et pour les autres ce sont des raisons de sécurité qui sont avancées (JRC, 2012). Ces réfugiés versent des bakchichs de 140 à 280 dollars (100 à $200 \mathrm{JD}$ ) aux employés syriens des deux postes frontières de Jaber et de Ramtha (JRC, 2012). Le passage par les points informels de Koya, Qusayr, Tal Shihab, Nasbeh, Tiba proches de Deraa, est plus dangereux, mais gratuit. Selon l'enquête, concernant 400 personnes, réalisée en 2012 par l'ONG italienne Un Ponte Per (2012 : 6), la moitié des Syriens serait entrée de manière légale jusqu'en juillet 2012, mais avec l'intensification des combats, les deux tiers franchirent la frontière par des passages illégaux. Dans l'urgence, avant l'ouverture des camps du HCR, deux petits camps dits " de transit " sont créés par le gouvernement en 2012 dans les terrains construits dans la zone franche d'Irbid encore non développée. Le King Abdullah Park regroupe ainsi 821 réfugiés fin 2013 dans une centaine de tentes (670 en juin 2015). Les réfugiés palestiniens de Syrie sont quant à eux logés dans un immeuble de six étages, prévu avant la crise économique pour devenir un pôle des technologies numériques, Cyber City. Ce bâtiment regroupe 300 réfugiés syriens mariés à des Palestiniens de Syrie et 202 réfugiés palestiniens de Syrie fin 2013 (respectivement 359 et 311 en juin 2015) ${ }^{11}$ (Hassan, 2014).

À partir de 2012, le HCR est présent et les réfugiés sont pris en charge par la police des frontières, nourris par les organisations caritatives islamistes (Takaful,

\footnotetext{
8 Seuls les frontaliers et les Syriens de classe moyenne possèdent des passeports. Avant la guerre, les Syriens devaient demander la permission de quitter le territoire, même avec un passeport valide.

9 Les principales confédérations tribales du Nord de la Jordanie, les Beni Khaled, Sardiya, Isa ont des membres vivant au sud de la Syrie. Et les familles Shraideh, Zubi, Rifai, Jaradat, Alawneh, Tibawi, Muqdad, Smadi et Zrayqat ont des membres des deux côtés de la frontière, animant le commerce transfrontalier. Leurs liens sont renforcés par des mariages fréquents entre Jordaniens et Syriennes, tradition qui remonte au moins aux années 1950 et qui s'est renforcée dans le contexte de crise depuis 2011.

10 Au début de la crise, les Syriens mettaient leurs familles à l'abri en Jordanie et retournaient travailler en Syrie, mais à partir de 2012, les passages sont devenus moins faciles et le paiement de bakchichs courants afin que les policiers jordaniens ne conservent pas les passeports.

11 UNHCR (2015) Factsheet, Field Office-Irbid, May-June 2015.
} 
Kitab wa Sunna et Islamic Relief) ${ }^{12}$, et dirigés vers les centres d'enregistrement du HCR à Ramtha et Amman, puis Irbid et Raba Sahran sont ouverts. Ils y déposent une demande pour bénéficier d'une carte de demandeur d'asile valide six mois, qui leur permet l'enregistrement auprès du ministère de l'Intérieur, afin d'obtenir une carte d'accès aux services médicaux et scolaires. La procédure prenait de six à huit mois au début de la crise, mais les temps ont été réduits à un à deux mois puis à quelques jours avec la multiplication des bureaux d'enregistrement à partir de février 2013. Jusqu'en décembre 2013, en infraction avec le droit international, la police des frontières conservait les originaux des documents syriens, que les réfugiés devaient réclamer pour retourner en Syrie. Les réfugiés obtenaient un reçu, qui ne leur permettait pas de remplir des demandes de contrat de travail ou d'enregistrer leurs actes d'état civil, mariages, naissances (Amnesty International, 2013). Mais sous la pression de plusieurs agences de protection, dont le HCR, le gouvernement a cessé ces pratiques, prétextes à bakchichs. Le HCR a aidé le gouvernement à classer 219462 passeports et pièces d'identité au cours de l'année 2014 et en a restitué 81603.

\section{Photo 1 : Classement des pièces d'identité des réfugiés syriens par le HCR}

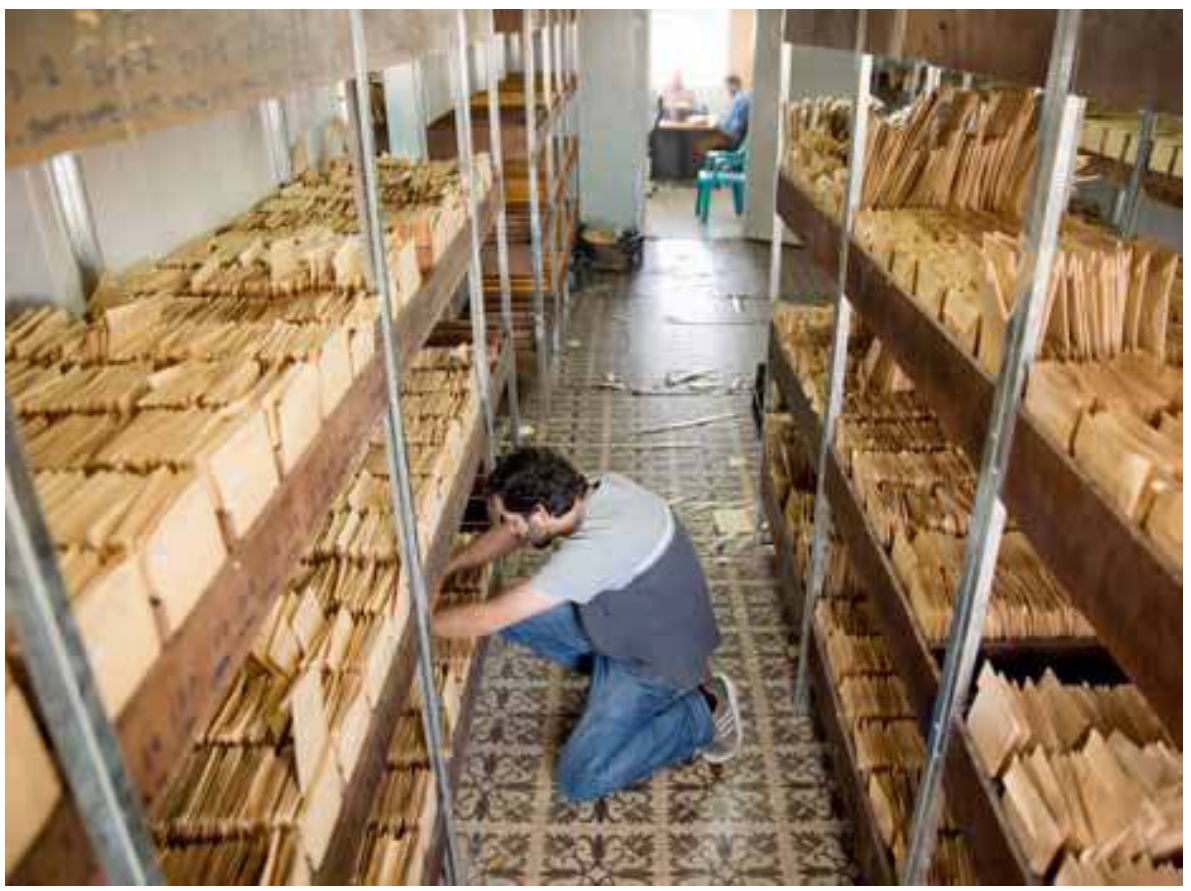

Source : UNHCR (2014) Year in Review. UNHCR Jordan.

12 Pour une description de ces ONG, voir UNHCR (2015a). Les organisations caritatives jordaniennes ont été les premières sur le terrain à apporter de l'aide aux " frères de Syrie ". Les comités de la zakat (aumône légale) présents dans chaque localité ont collecté les donations des fidèles via les mosquées (Hasselbarth, 2014 : 8). 
Tous les réfugiés syriens n'ont pas souhaité s'enregistrer auprès du HCR. Les procédures étaient longues et les cartes devaient être renouvelées d'abord tous les six mois, puis tous les ans en 2013. Selon UPP (2012 : 6), en 2012, 76,75\% des réfugiés syriens ont fait le choix de demander une carte de demandeur d'asile. $23,25 \%$ dit craindre de fournir des informations personnelles (c'est particulièrement le cas des combattants et des personnes torturées) ou avouent ne pas savoir comment s'enregistrer ou avoir du mal à se rendre à Amman pour le faire. Parmi l'ensemble des réfugiés les plus aisés estiment ne pas avoir besoin du statut de demandeur d'asile, d'autant qu'il empêche en théorie le retour vers la Syrie.

En septembre 2013, le HCR ouvre le centre d'enregistrement de Raba Sahran près de Mafraq, qui reçoit principalement des réfugiés qui ont franchi la frontière par les passages informels. Sept unités mobiles sont alors dispersées le long de la frontière pour les enregistrer. Ces passages, situés au sud de Deraa sont fermés l'été 2013 et seuls les blessés, militaires et civils, sont admis par Jaber. Les autres réfugiés sont alors obligés d'entrer en Jordanie en traversant le désert, au-delà de Ruweished, par les points de Rukban et Hadalat. À partir de juillet 2014, les Jordaniens contrôlent également les points de passage informels de la frontière orientale, situés en plein désert. Le rapport de Human Right Watch indique que des Syriens sont refoulés même après être arrivés au centre d'enregistrement de Raba Sahran, ce qui constitue une grave infraction au droit international sur les réfugiés (HRW, 2015).

Après les pics d'arrivée du printemps 2013 (30 000 par mois), la Jordanie ferme progressivement sa frontière et les flux s'effondrent à partir d'octobre 2013 à 20 000, puis 10000 entrées par an pour 2014 et 2015 (Figure 1). Ainsi que l'indique la figure 1, les renouvellements de cartes présentent trois pics : le premier autour d'avril 2014 s'explique par l'obligation de renouveler les cartes après un an. Le deuxième s'explique par la nouvelle obligation d'obtenir le permis de sortie des camps (bail out), qui entraîne la chute des renouvellements en juillet 2014, et le troisième, par l'ordre donné à tous les réfugiés de se réenregistrer après l'assassinat du pilote jordanien par l'El en février 2015. Notons qu'en l'absence de permis de sortie des camps, de nombreux réfugiés renoncent en 2015 à se réenregistrer, ce qui ne leur permet pas de scolariser leurs enfants et renforce leur vulnérabilité.

Figure 1 : Enregistrement des réfugiés syriens par le HCR en Jordanie depuis mars 2011

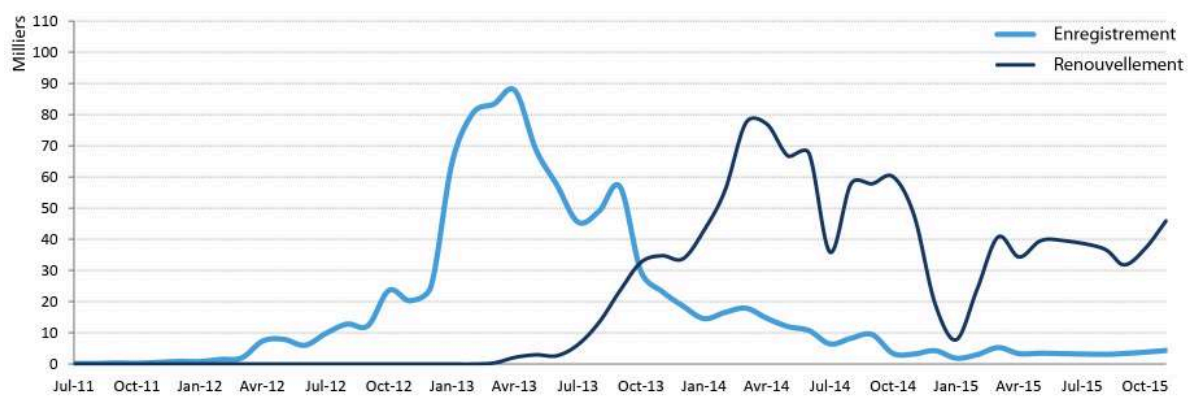

Source : UNHCR, External Statistical Report on Active Registered Syrians, 15 September 2015. Interagency Portal : http://data.unhcr.org/Syrianrefugees 


\section{3 : les réfugiés palestiniens de Syrie sont interdits d'entrée}

En dépit de son engagement à ne pas refouler les réfugiés, la Jordanie s'est autorisé le droit de filtrer les migrants qui demandaient l'asile, en refusant les hommes seuls n'ayant pas de preuve d'attachement familial dans le pays (de crainte qu'ils ne soient des combattants djihadistes), les personnes sans papier, ainsi que les réfugiés palestiniens et irakiens installés en Syrie. La raison invoquée concernant les réfugiés palestiniens de Syrie est la crainte d'un basculement démographique au profit des populations d'origine palestinienne, qui constituent déjà $45 \%$ de la population jordanienne. II s'agit de signifier à Israël que la Jordanie n'est pas la patrie de substitution de la Palestine (watan badil) ${ }^{13}$. Le gouvernement commença à refuser l'entrée des réfugiés palestiniens de Syrie en avril 2012, mais l'interdiction définitive fut prise en janvier 2013. En dépit de cette interdiction, environ 14000 réfugiés palestiniens de Syrie sont parvenus à entrer en Jordanie, seulement 1300 avant 2013 (HRW, 2014 ; Hassan, 2014). Ils sont parvenus à le faire en prétendant habiter dans les villages du gouvernorat de Deraa et en utilisant des réseaux de passeurs. Certains ont été refoulés en Syrie, ce qui est contraire aux résolutions internationales sur la protection des réfugiés. Les réfugiés palestiniens de Syrie sont particulièrement vulnérables ${ }^{14}$. Ils ne peuvent pas résider dans les camps du HCR et doivent travailler dans des conditions difficiles pour payer leurs loyers dans les villes et villages jordaniens. 180 Palestiniens furent hébergés par le gouvernement jordanien dans le complexe de nouvelles technologies Cyber city construit pour la ville d'Irbid à la fin des années $2000^{15}$. Les conditions de résidence sont quasi carcérales avec deux postes de police qui contrôlent les entrées et les sorties des employés des organisations humanitaires. Les réfugiés ne peuvent sortir du camp que pour rentrer en Syrie, ou pour rendre visite à leurs proches installés dans des villes jordaniennes, mais pour une période de quarante-huit heures seulement chaque semaine (Hassan, 2014).

\section{4 : démantèlement des camps informels et interdiction d'entrer par voie aérienne}

La Jordanie prône une politique de frontière ouverte et dit accepter et recevoir tout réfugié blessé et leurs familles. Mais dans les faits, depuis 2014, les quarante-cinq points de passage illégaux sont désormais inutilisables, des barrières ayant été érigées, ce qui contraint les réfugiés à marcher vers l'Est, à une semaine de marche dans le désert contrôlé par l'armée syrienne, vers les postes de Rukban et Hadalat, situés à cent et cinquante kilomètres de Ruweished (Carte 1). À partir de l'été 2014, les flux de réfugiés s'effondrent, du fait de la multiplication des combats près de la frontière syrienne. Des milliers de réfugiés se trouvent bloqués dans le no man's land situé côté jordanien, officiellement parce que le régime syrien interdirait les passages, mais officieusement parce que la Jordanie a décidé de filtrer les réfugiés. En avril 2015, la Jordanie ferme

\footnotetext{
13 Jordan News Agency (Petra), PM: Jordan Will Not Be a Substitute Homeland for Palestinians, 26/02/2014 (Hassan, 2014).

14 Voir l'article de Kamel Doraï dans ce numéro.
}

15 Le problème se posa alors des citoyens jordaniens d'origine palestinienne résidant en Syrie, qui furent considérés comme des réfugiés palestiniens et furent, pour dix d'entre eux, privés de leur nationalité et renvoyés en Syrie (HRW, 2014). 
son poste frontière de Jaber (Nassib) trop exposé aux incursions de djihadistes de Jabhat Al-Nusra et de I'El.

À partir de 2014, les mesures de contrôle des réfugiés en milieu urbain se sont accrues. Tout d'abord, les autorités ont entrepris de démanteler les 125 camps informels de tentes installés dans la vallée du Jourdain et dans les villages du gouvernorat de Mafraq, près des terres agricoles et des serres. Ces camps regroupaient plus de 10000 personnes et se déplaçaient suivant le calendrier agricole (REACH, 2014c) (Photo 2). En mai 2014, un camp informel de tentes de 1000 réfugiés syriens, à Sahab au sud-est d'Amman a été démantelé en quelques heures par un escadron de 1000 hommes armés ${ }^{16}$. Un tel déploiement de force était dû à la présence de combattants parmi les hommes installés dans ce camp, situé à l'un des carrefours routiers vers I'Irak.

\section{Photo 2 : Camp informel sur le terrain d'un particulier}

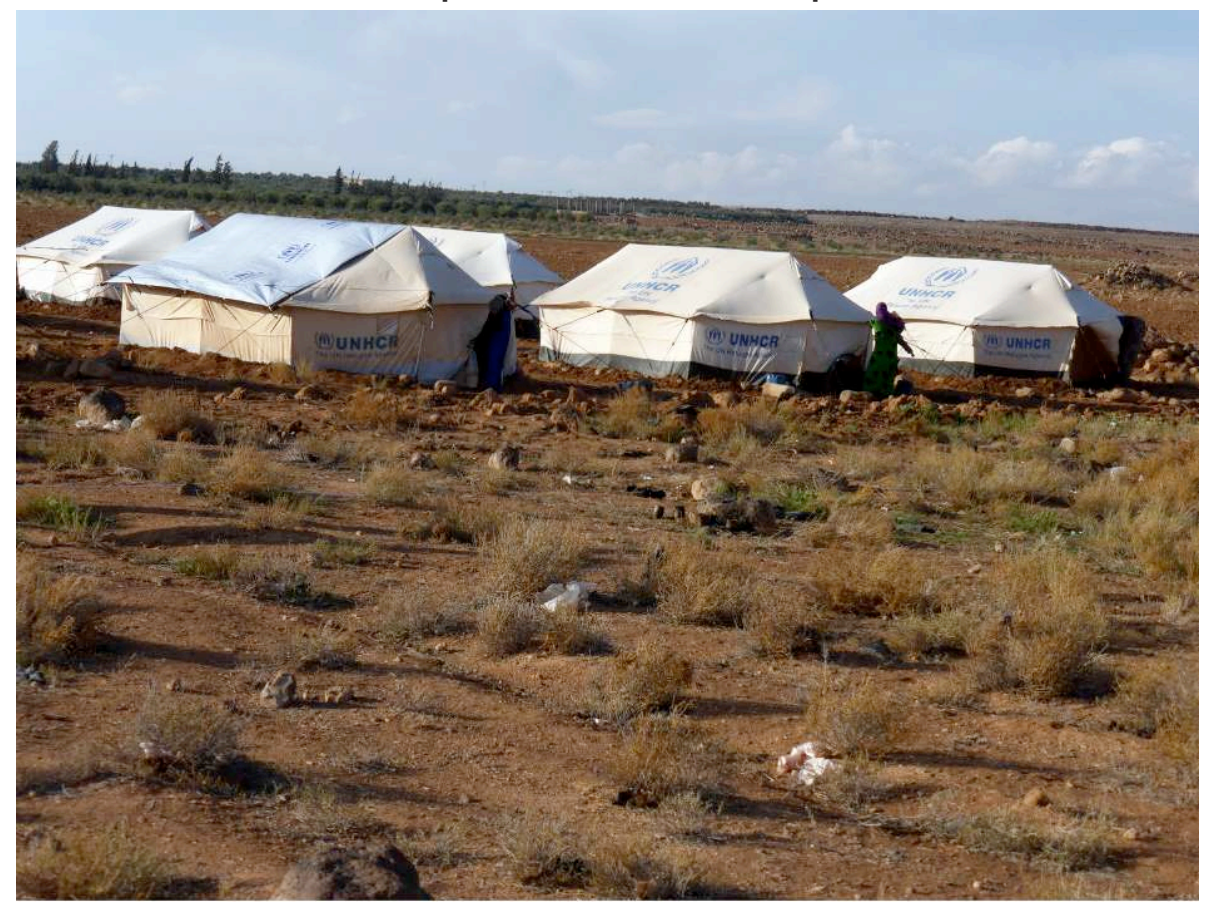

Crédit : M. Ababsa, mars 2014.

En mai 2014, une nouvelle étape est franchie dans le contrôle des réfugiés syriens quand l'interdiction d'entrer par l'aéroport d'Amman est prise pour les non-détenteurs de permis de résidence jordanien. II s'agit pour les autorités de contrôler les flux de combattants entre la Syrie, la Jordanie, I'Irak et la Libye. II s'agit aussi de préserver les touristes arabes et occidentaux. En revanche, les

16 UNHCR Jordan, Protection Working Group Meeting minutes, may 2014. La ville de Sahab est avec Maan, I'une des plus dangereuses du pays pour sa criminalité du fait de sa situation de carrefour routier, mais aussi par la présence de réseaux de trafiquants. 
réfugiés syriens présents sur le territoire ont le droit de prendre l'avion, mais uniquement pour se rendre en Turquie (pays qui n'exige pas de visa pour les Syriens).

Selon le gouvernement jordanien, environ 90000 réfugiés seraient retournés en Syrie en 2013, mais selon le HCR une grande partie serait revenue (UNHCR, 2014 : 4). Ils sont de plus en plus nombreux à le faire depuis la fin août 2015, avec la possibilité d'être accueilli en Europe. En juillet 2015, une moyenne de soixante-six réfugiés quittait le camp chaque jour, ce chiffre est passé à 129 par jour en août, 300 par jour en octobre 2015 (UNHCR-ISWG, 2015 ; Oddone et Reznick, 2015). Une partie est acheminée par la police des frontières au-delà de Tel Shihab ${ }^{17}$. En majorité, ces Syriens tentent de regagner leur village, ayant épuisé toutes leurs ressources. Seule une petite partie de ces migrants parvient à rejoindre la Turquie à travers la Syrie en guerre, au péril de leur vie. Selon une enquête conduite par le HCR auprès de 600 familles de réfugiés en septembre 2015, 7 \% disent vouloir rentrer en Syrie et $18 \%$ disent vouloir atteindre la Turquie dans les trois mois (UNHCR-ISWG, 2015).

\section{La menace djihadiste, la sécurisation des frontières et le contrôle des réfugiés}

L'intensification des combats en Syrie et la situation en Libye en 2014 et 2015 conduisent le régime à durcir sa politique. La loi antiterroriste de 2006 est complétée en avril 2014 par une loi plus restrictive qui muselle les sites d'opposition et les journalistes trop critiques ${ }^{18}$. Devant la menace de l'État islamique et dès le printemps 2014, les Jordaniens sécurisent les deux frontières le long de la Syrie (378 kilomètres) et de I'Irak (175 kilomètres) au moyen de tranchées, de barrières et de tours de garde financées par les États-Unis. En septembre 2015, Israël entreprend à son tour de sécuriser la frontière avec la Jordanie.

Après I'assassinat du pilote jordanien Moath Al Kassabeh par l'État islamique en Syrie en janvier 2015, le gouvernement a ordonné en février 2015 une vaste opération de vérification de l'identité des réfugiés syriens et le renouvellement de toutes les cartes de résidence. Cette opération qui s'est accompagnée de la prise de photographies de l'iris de l'œil a des visées sécuritaires ; des combattants islamistes s'étant mêlés aux réfugiés, selon le gouvernement jordanien ${ }^{19}$. Les réfugiés doivent fournir des certificats médicaux (13 JD par personne), ainsi qu'un contrat de location, taxé à $10 \%$ du montant du loyer. Pour une famille de cinq, la taille moyenne des ménages, cette opération coûte environ 85 JD (120 US\$), ce qui représente une lourde somme pour la majorité des familles ${ }^{20}$.

17 Conversation avec un haut fonctionnaire du bureau jordanien du HCR à Amman, 14 octobre 2015.

18 Cf. URL : http://www.aljazeera.com/news/middleeast/2014/04/jordan-anti-terrorism-lawsparks-concern-201442510452221775.html et https://freedomhouse.org/report/freedomworld/2014/jordan

19 Les réfugiés qui n'ont pas renouvelé leurs cartes de résidence sont conduits vers le nouveau camp d'Azraq.

20 Entretiens avec des réfugiés syriens à Irbid en février 2015. 


\section{La politique de cantonnement dans les camps de réfugiés}

Dans le but de mieux organiser l'assistance humanitaire et de contrôler les populations de migrants forcés, la Jordanie a souhaité diriger les réfugiés syriens dans de vastes camps administrés par le HCR ${ }^{21}$. Cette politique de création de camps prend tout son sens au regard du traitement de la crise irakienne dix ans auparavant. Durant les années 2003-2008 les 200 à 300000 réfugiés irakiens arrivés en Jordanie après I'intervention américaine, s'étaient rapidement fondus dans le paysage urbain, rejoignant des membres de leur famille installés dans les années 1990, si bien que le gouvernement jordanien avait eu des difficultés à obtenir le soutien financier des bailleurs internationaux. Le manque de visibilité de l'aide avait été rédhibitoire et les autorités avaient dû gonfler les chiffres de réfugiés afin de justifier l'ouverture d'écoles supplémentaires pour accueillir les élèves irakiens (Seeley, 2010 ; Chatelard, 2009).

Dans le cas des réfugiés syriens, l'implantation de camps relève de deux objectifs politiques. Le premier est sécuritaire, les camps permettant d'enfermer les réfugiés hors de l'espace public jordanien, en particulier pour le camp d'Azraq, vers lequel le gouvernement a entrepris en 2014 de diriger les réfugiés arrêtés en situation irrégulière (carte de résidence périmée, absence d'autorisation de sortie des camps - bail out - ou délit de travail, les réfugiés n'ayant pas le droit de travailler). Le second consiste à montrer à la population jordanienne que le pays sait gérer une crise humanitaire sans réduire les services scolaires, médicaux, ainsi que les infrastructures de base destinés aux Jordaniens.

La Jordanie gère les réfugiés syriens dans trois types de camps de réfugiés différents : de petits " camps de transit " ouverts en 2012 et encore en fonction (King Abdullah Park et Cyber City près d'Irbid), des camps de réfugiés administrés par le HCR (Zaatari et Azraq) et par le Croissant rouge émirien (Mrajeeb Al Fhood) et enfin, les camps de rétention situés près de la frontière, en contravention avec les droits fondamentaux des migrants. Au 15 septembre 2015, 109391 réfugiés syriens vivent dans les camps situés dans les gouvernorats de Mafraq et de Zarqa : 79433 réfugiés syriens à Zaatari, 23622 à Azraq et 6336 dans le camp émirien ${ }^{22}$. Alors que Zaatari ne cesse de perdre des habitants, les camps d'Azraq et du croissant rouge émirien en gagnent depuis 2014 (Figure 2).

21 Le pays comptait déjà en 2011 treize camps destinés aux réfugiés palestiniens, dix gérés par I'UNRWA et trois par le gouvernement. Ces camps qui comptent $20 \%$ de l'ensemble des réfugiés palestiniens sont considérés comme symbole du maintien du droit au retour (Al Husseini, 2011).

22 UNHCR (2015) External Statistical Report on Active Registered Syrians, 15 September 2015. 
Figure 2 : L'évolution de la population des camps de réfugiés syriens de Jordanie depuis avril 2014

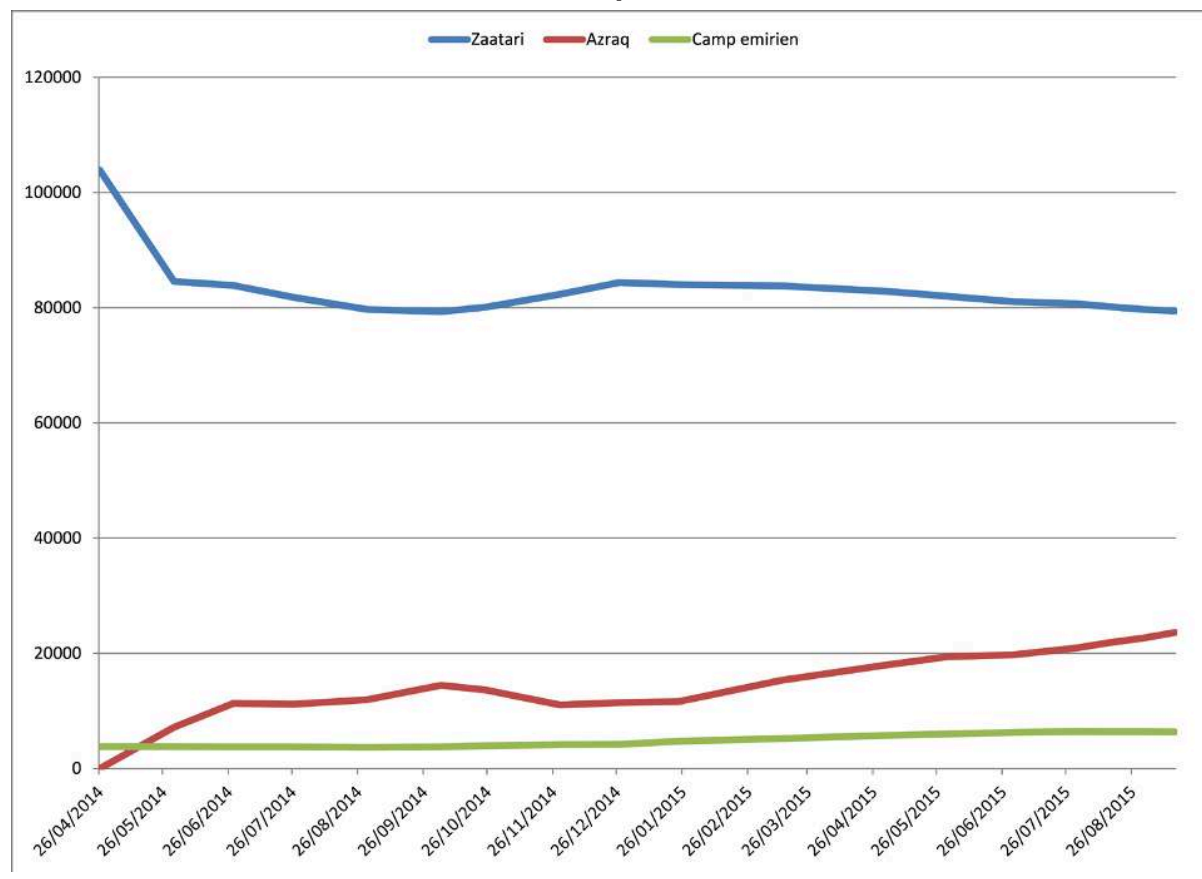

Source : UNHCR, External Statistical Report on Active Registered Syrians, 15 September 2015.

\section{Le camp de Zaatari (UNHCR)}

En juillet 2012, le HCR et le gouvernement ouvrent le camp de réfugiés de Zaatari, dans le gouvernorat de Mafraq, dans la steppe (badia), sur la route de I'Irak. II est tout d'abord co-administré par le HCR et le Jordan Hashemite Charity Organisation (JHCO), dont la mission est de coordonner l'ensemble de l'assistance fournie par les ONG internationales et les donateurs internationaux. À partir de mars 2013, le Syrian Refugee Camp Directorate, dépendant du ministère de l'Intérieur, le prend en charge, du fait des nombreux problèmes liés à la sécurité, au vandalisme et à violence. Le camp était alors la quatrième ville du pays, avec 140000 habitants et le deuxième camp de réfugiés au monde. Beaucoup de réfugiés ont quitté le camp, en raison de la présence de criminels de droit commun syriens sortis de prison, de gangs terrorisant la population, de combattants qui ont importé les conflits politiques internes entre les membres de l'armée syrienne libre, les supporters du Conseil national syrien, les membres de la Coalition nationale de l'opposition et ceux du Conseil tribal syrien, ainsi que les djihadistes fidèles à Jabhat al-Nusra, (UNHCR, UNICEF et WFP, 2014) ${ }^{23}$. 
Progressivement, le gouvernement et le HCR mirent sur pied toute une organisation de médiation, via des leaders tribaux ou des chefs de famille, aidés en cela par une certaine homogénéité tribale des réfugiés de Zaatari et ont écarté les agitateurs et les chefs de gangs. Malgré ces mesures, des difficultés subsistent, en particulier l'éloignement des unités sanitaires qui les rend dangereuses la nuit pour les femmes seules avec notamment l'absence de poste de police. Une économie parallèle s'est développée et des commerces installés le long de la rue centrale, nommée par dérision les Champs-Élysées, se sont branchés illégalement sur le réseau du HCR et ne paient pas l'électricité ${ }^{24}$. Le HCR a introduit un programme de travail pour les réfugiés qui le souhaitent (cash for work) : ils reçoivent de 1 à 2 JD par heure selon leur niveau de compétence.

Photo 3 : Réfugiés circulant au milieu du camp de Zaatari

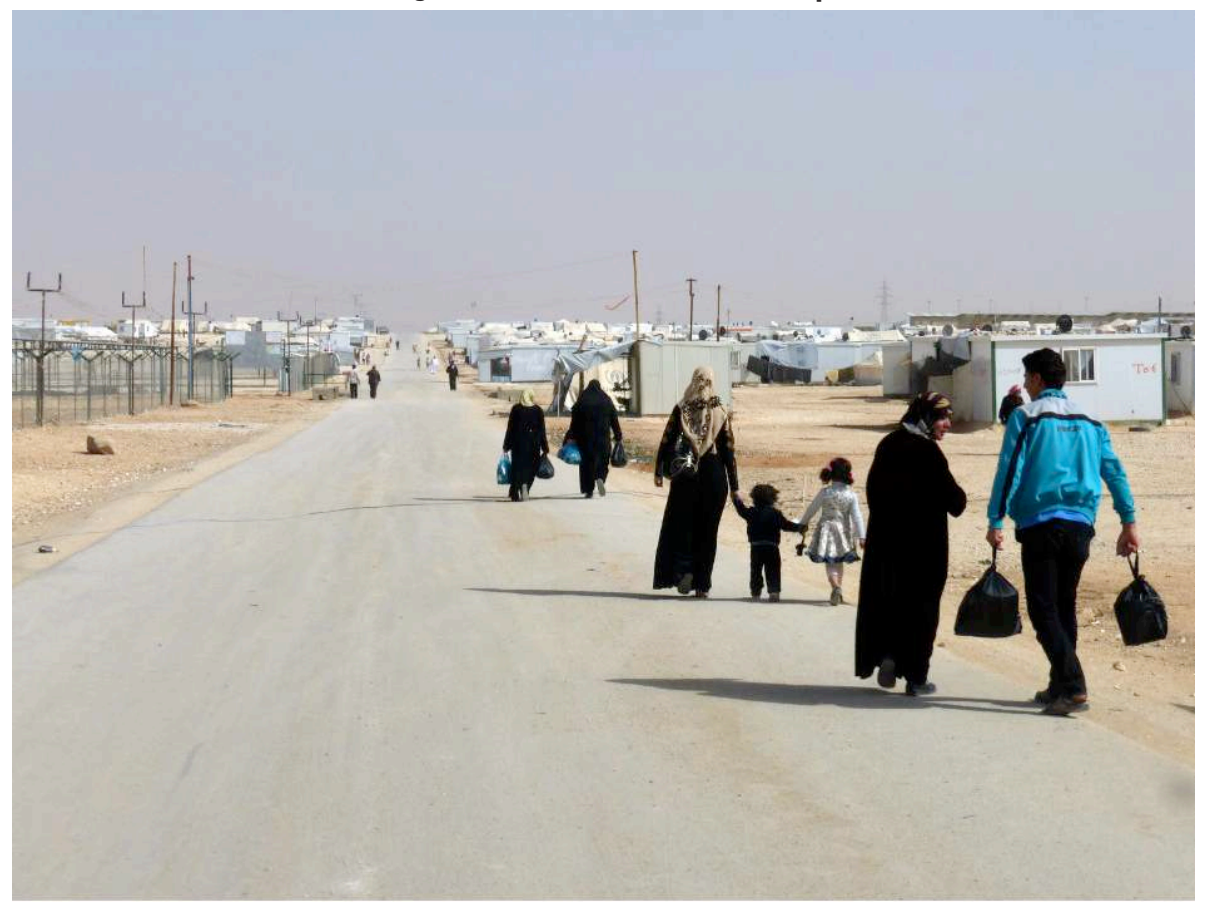

Crédit : M. Ababsa, mars 2014.

Près de 350000 réfugiés ont transité par Zaatari depuis 2012, les autorités dirigeant vers ce camp ceux qui avaient traversé la frontière de façon illégale (sans tampon d'entrée sur leur passeport). Mais la population du camp de Zaatari n'a cessé de se réduire depuis 2013, les réfugiés préférant chercher un travail hors du camp. Une opération de vérification de l'identité des réfugiés du camp de Zaatari en janvier et mai 2014, afin de leur " restituer leurs documents ", a eu pour conséquence de chasser encore plus de personnes hors du camp. 
Photo 4 : Les « Champs Élysées », rue principale du camp de Zaatari

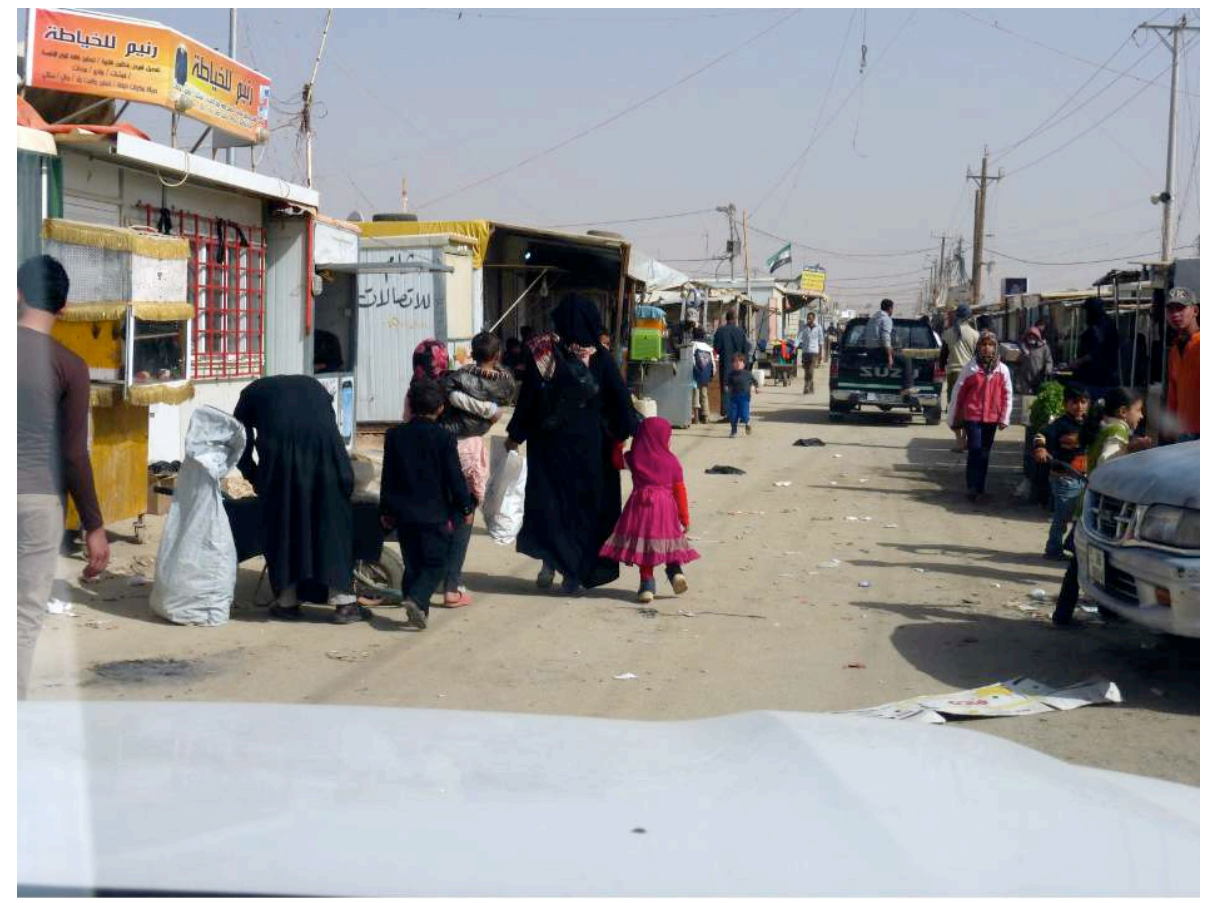

Crédit : M. Ababsa, mars 2014.

\section{Le camp d'Azraq}

La construction du camp d'Azraq fut décidée par le gouvernement jordanien dès 2013 , alors que le camp de Zaatari était saturé. Ce nouveau camp prévu pour 52000 réfugiés peut atteindre 100000 personnes si nécessaire. II a ouvert en avril 2014, sur un terrain basaltique aride, quatre fois plus vaste que le camp de Zaatari, à vingt kilomètres au sud de la petite ville d'Azraq (5 000 habitants). Les architectes disent avoir tiré les leçons du camp de Zaatari pour l'aménager : le camp d'Azraq est entouré d'une barrière et d'un fossé. Chacun des huit villages, prévus pour 12500 personnes, dont quatre sont opérationnels et habités en 2014 est structuré autour d'un poste de police et de services médicaux sanitaires ont été conçus pour être accessibles à un nombre réduit de préfabriqués. Les préfabriqués (" abris en T ») sont conçus avec des matériaux isolants, mais sont recouverts de tôles blanches qui éblouissent. Ils ne comportent qu'une seule fenêtre qui ne permet pas l'aération correcte du logement. De façon générale, les conditions climatiques sont très dures et l'électricité ne sera installée que fin 2015.

Le camp d'Azraq a vu sa population passer de 14453 en octobre 2014 à 11441 en décembre 2014. Les témoignages font état des difficiles conditions de vie et de la dépression des réfugiés qui y arrivent, d'autant que nombreux sont ceux qui sont conduits au camp de force par la police. Depuis 2015, la police jordanienne envoie au camp d'Azraq les réfugiés arrêtés alors qu'ils mendient 
(la mendicité étant interdite) ou qu'ils travaillent de façon illégale. En 2014, 8500 réfugiés ont été dirigés contre leur gré vers le camp d'Azraq (NRC, 2015). La politique est si efficace qu'en un an, la population du camp d'Azraq a doublé, passant à 23622 en septembre $2015^{25}$.

Photo 5 : Toilettes du camp d'Azraq avant son ouverture

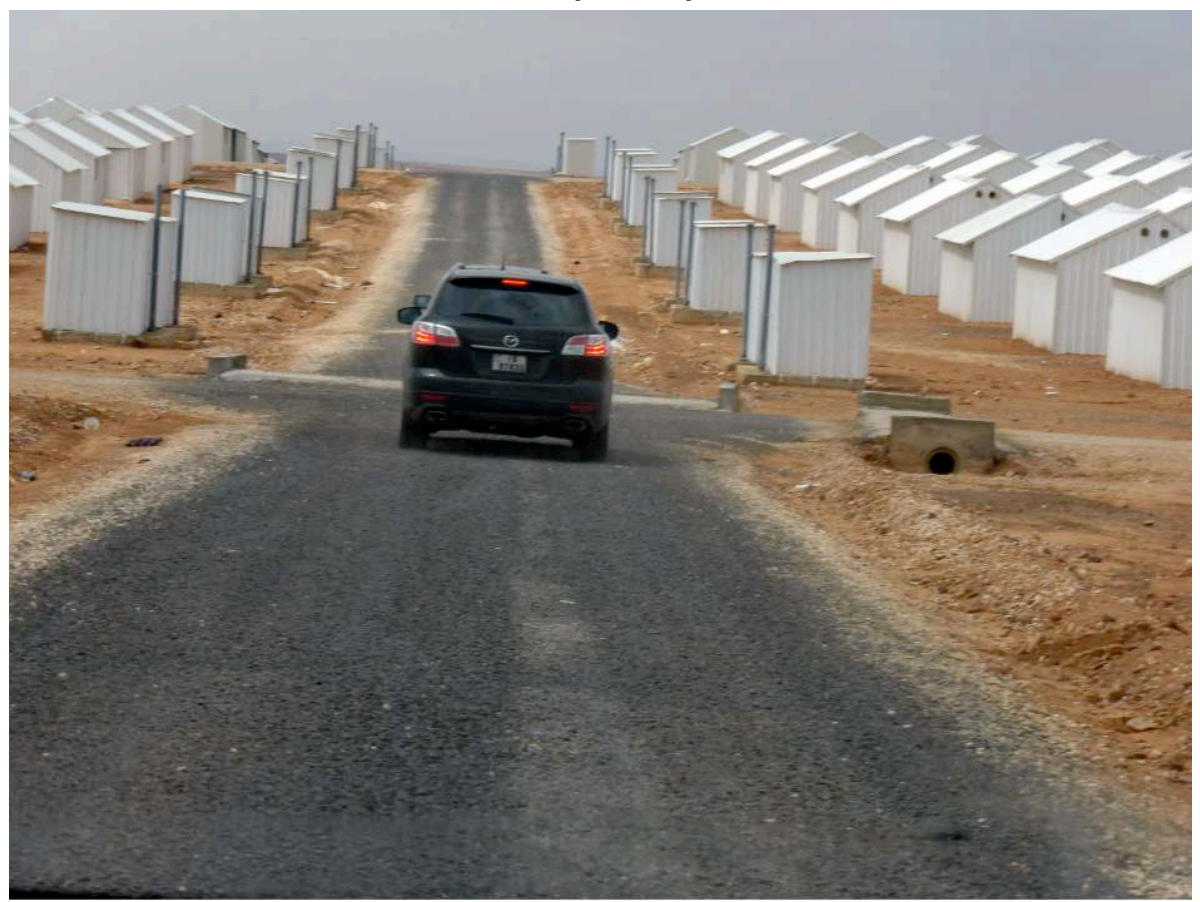

Crédit : M. Ababsa, mars 2014.

\section{Le " camp cinq étoiles " du Croissant rouge émirien (Mrajeeb Al Fhood)}

Le camp est situé à Mrajeeb Al Fhood, à vingt kilomètres de Zarqa. Sa population a doublé en un an, passant de 3746 personnes à 6433 en août 2015. Son coût de fonctionnement est très élevé, se montant à 15 millions de dollars pour 4000 réfugiés en deux ans. II témoigne de la volonté des Émirats de montrer leur générosité en faveur des coreligionnaires syriens. Le camp n'est ouvert qu'aux familles et refuse les hommes seuls. Mais les conditions de sélection ne sont pas claires et alimentent la rumeur selon laquelle les femmes y seraient plus nombreuses. C'est un espace sécurisé, clôturé de fils barbelés, et dont les réfugiés ne peuvent sortir qu'au compte-goutte, pour des périodes de trois jours seulement. Ce "camp cinq étoiles ", ainsi nommé par les Syriens, offre des conditions de résidence confortables, dans des logements préfabriqués ${ }^{26}$

25 UNHCR (2015) External Statistical Report on Active Registered Syrians, 15 September 2015.

26770 en octobre 2013, 800 de plus en avril 2015 (Irin, 2013). 
équipés de salles de bain et d'eau courante, ce qui permet aux familles de ne pas exposer les jeunes filles aux dangers des toilettes publiques. Ils sont équipés de ventilateurs, de réfrigérateurs, de télévision et antenne parabolique. Les réfugiés, qui n'ont pas l'autorisation de cuisiner, bénéficient de trois repas par jour avec viande et fruits, régime alimentaire que ni les Syriens ni les Jordaniens ne connaissent, étant donné le coût de ces denrées. Thé et café sont offerts à volonté dans les centres sociaux climatisés, offrant des écrans de télévision, mais séparés selon le sexe. Enfin, les réfugiés peuvent choisir parmi les luxueux vêtements offerts par les Émiriens (IRIN, 2013).

En remerciement de sa générosité, le gouvernement laisse les Émirats arabes unis gérer le camp et ne lui fournit qu'un soutien policier. Un homme par famille peut travailler au sein du camp dès lors qu'il s'inscrit selon ses compétences sur les listes d'emplois vacants. La plupart s'occupent des tâches de collecte des ordures et de nettoyage, mais ils peuvent également rejoindre l'atelier artisanal, qui construit les meubles des principaux bâtiments publics, notamment une mosquée installée sous une tente climatisée. Ces hommes reçoivent ainsi en moyenne 25 JD par semaine. Une clinique assure des soins de médecine, de pédiatrie, d'obstétrique, de stomatologie, de radiologie ainsi qu'un service de garde, assurés en partie par les réfugiés qui sont médecins. Une école gérée par I'UNICEF pour 1300 élèves a ouvert en septembre 2013, avec le soutien financier de la coopération britannique.

Photo 6 : Le camp de réfugiés syriens géré par les Émirats

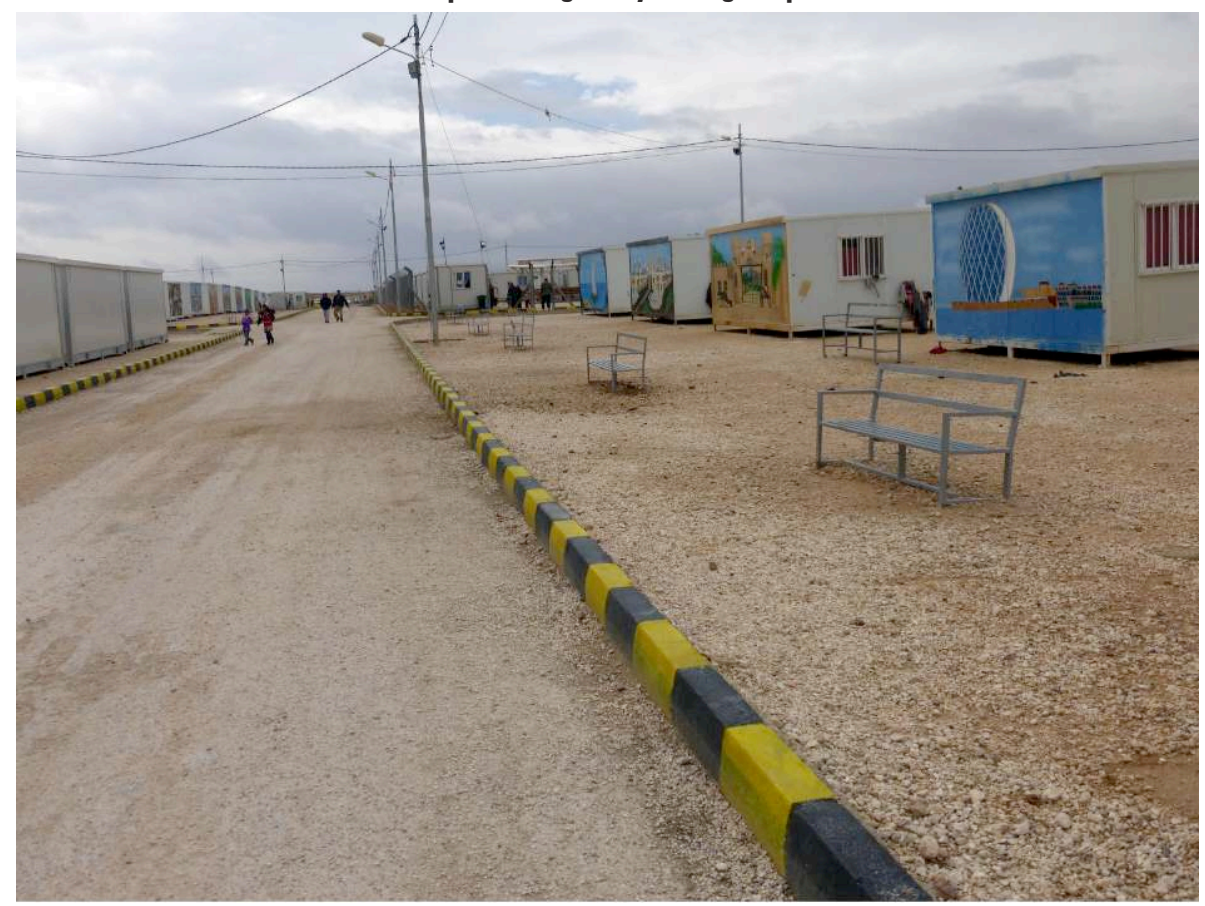

Crédit : M. Ababsa, mars 2014. 


\section{Permis de sortie et renouvellement des cartes de demandeurs d'asile}

Les réfugiés installés dans les camps de Zaatari et d'Azraq ne sont autorisés à sortir qu'après avoir obtenu un permis (bail out) et l'attestation d'un Jordanien qui se porte garant (kafil), payé 100 à 300 JD. Ce permis émane du Département d'Assistance aux Réfugiés Syriens (Syrian Refugee Assistance Department SRAD). Mais depuis avril 2014, date de l'ouverture du camp d'Azraq, le SRAD est de plus en plus réticent à donner ces permis, que ce soit pour le camp de Zaatari ou celui d'Azraq. Désormais, les permis ne sont accordés qu'à des kafils qui sont réellement liés à la famille par des liens de sang ou matrimoniaux.

En outre, depuis le 14 juillet 2014, les autorités jordaniennes exigent du HCR qu'il ne renouvelle plus les cartes de demandeurs d'asile des réfugiés de ceux qui ont quitté les camps sans autorisation de sortie (bail out). Or cette mesure a de graves conséquences puisque les réfugiés ne peuvent plus obtenir de cartes de service du ministère de l'Intérieur et n'ont pas accès aux écoles et aux soins gratuits. Dans les faits, les directeurs d'école sont conciliants et laissent les enfants syriens assister aux cours, avec ou sans carte de service, et sans certificat de naissance ni de niveau, nécessaire à toute inscription. Le ministère de l'Éducation prend ainsi ses distances avec le ministère de l'Intérieur, en étant fidèle à sa mission d'enseignement de l'ensemble des enfants présents sur le territoire (INEE et ESWG, 2015).

\section{Les espaces de rétention de Rukban et de Hadalat}

À partir de juillet 2014, les autorités jordaniennes entreprennent de filtrer les réfugiés qui entrent par les points de Rukban et Hadalat. II s'agit de s'assurer que des djihadistes venus des zones sous contrôle de I'El, Deraa et Raqqa, ne se mêlent pas aux flux de réfugiés. Les réfugiés sont alors bloqués sur un talus de sable (" berm " en anglais), qui marque la frontière, entouré de fils barbelés. Ils se sont construit des abris à partir de bâches et d'anciennes tentes près de Rubkban. En octobre 2014, environ 4000 réfugiés y étaient bloqués. En octobre 2015, ils sont encore 2000. Ils ne reçoivent de l'aide que de la Croix-Rouge internationale, alors que le HCR demande instamment qu'ils soient transférés dans le camp d'Azraq. Entre novembre et mars 2015, le camp a été démantelé et les organisations humanitaires, dont l'Organisation Internationale des Migrations (OIM), ont accompagné les réfugiés vers le centre d'enregistrement de Raba Sahran, puis vers le camp d'Azraq. Mais en avril 2015, après I'assassinat du pilote jordanien en Syrie, deux nouveaux camps de rétention se sont reconstitués sur le berm, I'un comptant 175 tentes près de Rukban et l'autre, 68 tentes près de Hadalat, abritant environ 2500 réfugiés (HRW, 2015).

Mais, la majorité des réfugiés vit hors des camps, au sein des villes et des villages jordaniens, louant à prix très élevés des logements modestes, voire délabrés. Ils sont accusés d'exercer une pression accrue sur les infrastructures d'approvisionnement en eau, de collecte des ordures, ainsi que sur les services scolaires et médicaux. 


\section{L'aggravation des conditions de vie des réfugiés syriens hors des camps}

L'arrivée des réfugiés syriens s'est produite dans une situation d'après crise économique et alors que de nouvelles zones franches s'ouvraient pour aider à la création d'emploi. Aussi les citoyens jordaniens les plus démunis ont vu avec une exaspération croissante les réfugiés syriens leur faire concurrence tant pour le logement, que pour l'accès aux services publics et au marché de l'emploi informel. Ces récriminations ont culminé lors des manifestations de novembre 2012, consécutives à la réduction des subventions sur le fuel et l'électricité, dont " trop de non-Jordaniens bénéficiaient ". Cette incrimination des réfugiés alors que des raisons géopolitiques (les actes de terrorisme sur les gazoducs venant d'Égypte ${ }^{27}$ ) avaient présidé à cette décision, permet de saisir le contexte de tension croissante. Le déficit budgétaire jordanien ne cesse de s'alourdir et la réduction du commerce international par la Syrie vers la Turquie a eu un impact très négatif sur l'économie. En 2013, le gouvernement a contracté un emprunt de 2 milliards de dollars au FMI pour combler son déficit de 2,8 milliards. En 2014, il annonce que le coût de la présence des réfugiés syriens est de 871 millions de dollars, soit 2,4\% du PNB, et réclame une assistance à hauteur de 1,2 milliard de dollars (Jordan National National Resilience Plan 2014-2016). L'économiste Yusuf Mansur, devenu le directeur de la zone économique spéciale d'Aqaba, considère que ces réfugiés dynamisent le pays par leur seule présence de consommateurs, sans compter leurs compétences et leur argent. L'économiste fut le premier à citer le chiffre d'1 milliard d'investissement apporté par les Syriens en 2013, selon le Jordanian Investment Board (Dhingra, 2014). Le chiffre a, par la suite, été repris. En comparaison, en 1990, le retour des Palestiniens du Golfe aurait insufflé 1,6 milliard de dollars à l'économie jordanienne ${ }^{28}$.

Si les premiers migrants avaient bénéficié des réseaux de solidarité familiale et de l'aide caritative des ONG jordaniennes, au fil des années, les réfugiés ont épuisé leurs maigres ressources et sont obligés de chercher du travail dans la construction, l'agriculture et les petits commerces et de vendre les coupons alimentaires et les objets qui leur sont distribués. $86 \%$ des réfugiés vivent sous la ligne de pauvreté de 68 JD par mois et par personne (UNHCR, 2015b). En dépit d'une politique active de scolarisation des enfants et de la campagne No Generation Lost menée par UNICEF, moins des deux tiers des enfants syriens sont scolarisés (60\% en 2014 et autour de la moitié en 2015). La conséquence de l'arrivée des réfugiés syriens a été une pression accrue sur les infrastructures présentes, déjà déficitaires. Les loyers ont souvent doublé dans les gouvernorats du Nord du pays où se concentrent les réfugiés. Alors que les revenus des réfugiés s'effondrent, avec la réduction de I'aide humanitaire et en particulier des coupons alimentaires distribués par le Programme Alimentaire Mondial (PAM), I'assistance humanitaire islamique prend une importance croissante.

27 La Jordanie importe $96 \%$ de ses besoins énergétiques pour un coût équivalent à $16 \%$ du PIB en 2013. Jusqu'en 2003, les centrales électriques jordaniennes fonctionnaient au fuel irakien à très bas prix. À partir de 2003, les centrales ont fonctionné au gaz importé d'Égypte à prix préférentiel. Mais une série d'attaques terroristes contre les gazoducs venant d'Égypte en février 2011 a contraint le gouvernement à importer du fuel à prix élevé d'Irak. En conséquence, la levée des subventions sur les hydrocarbures fut adoptée en novembre 2012.

28 MansurYusuf (2013) The cost of refugees, Jordan Times, 04/02/2013 http://www.jordantimes.com/opinion/yusuf-mansur/cost-refugees 


\section{Plus de $80 \%$ des réfugiés syriens de Jordanie vivent en milieu urbain}

Les réfugiés constituent plus de la moitié de la population du gouvernorat de Mafraq, dont la petite ville a été très dynamisée par des centaines de nouveaux commerces. En quête de logements à louer, les Jordaniens ont bâti à la hâte des constructions de fortune et réaménagé des garages. Ils les louent aux réfugiés qui se regroupent à deux ou trois familles par appartement, afin de partager les loyers (Figure 4). L'idée répandue est que les Syriens peuvent payer, car ils sont aidés par les organisations internationales. Dans les faits, aucune ONG ne contribue aux paiements des loyers et les réfugiés doivent se débrouiller en vendant notamment les coupons alimentaires qu'ils reçoivent. Pour la moitié des réfugiés, les loyers s'élèvent à plus de 150 JD par mois, de deux à trois fois supérieurs à ce que les Jordaniens payent. Ils représentent $57 \%$ de leurs dépenses (UNHCR, 2014) et $10 \%$ des réfugiés sont menacés d'expulsion (NRC, 2015). Le dernier rapport de Care a montré qu'en 2015, le niveau moyen des loyers demandés aux Syriens a baissé de $16 \%$ en raison de l'insolvabilité progressive de ces derniers (Care, 2015).

Figure 3 : Loyers moyens payés par les réfugiés syriens en Jordanie

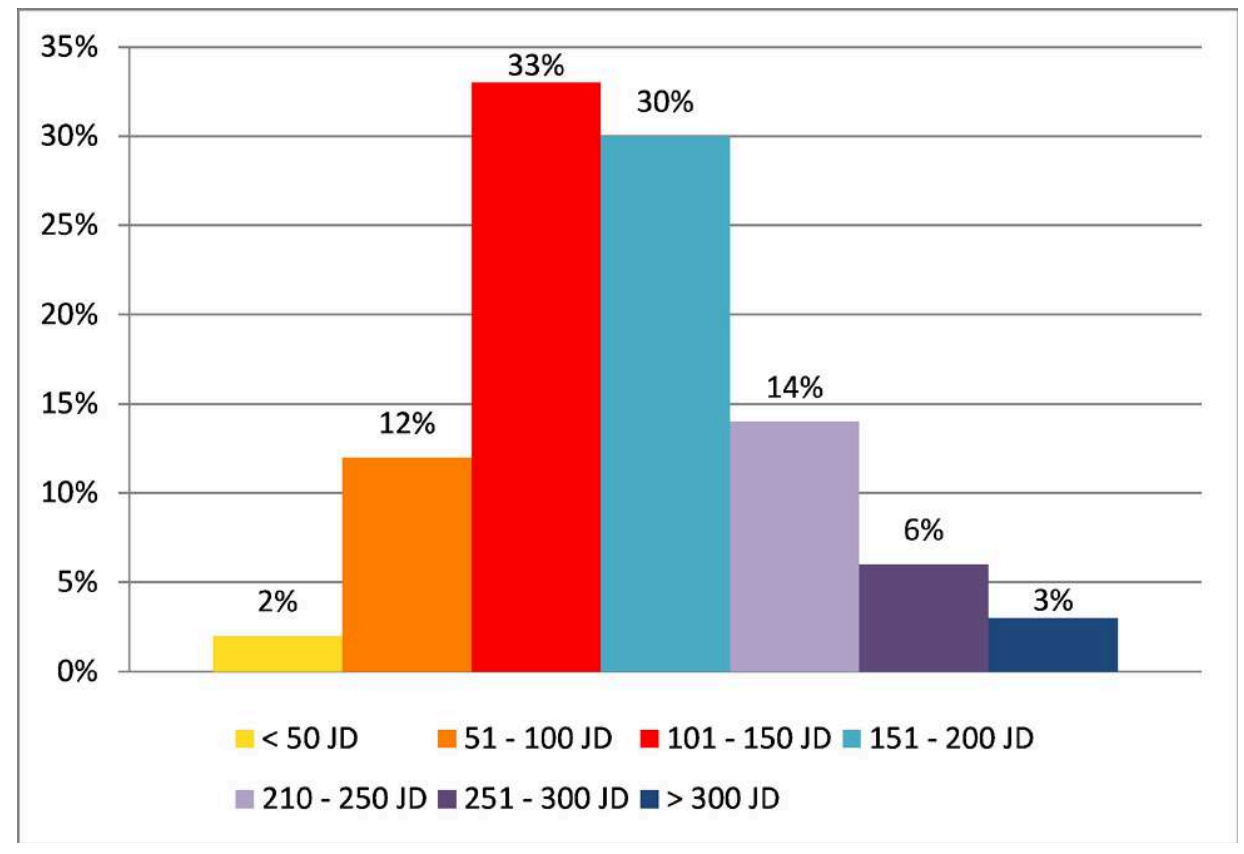

Source : NRC 2015, 4964 cas, 31/12/2014. 
Figure 4 : Composition des ménages de réfugiés syriens

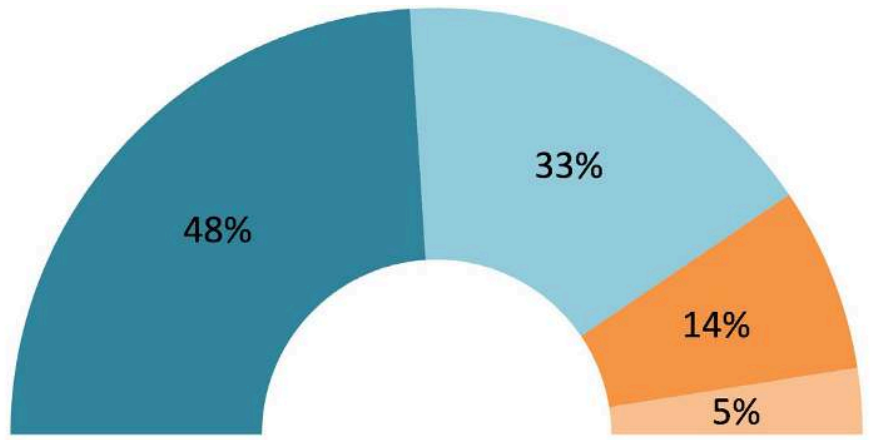

1 famille $\quad 2$ familles $\square 3$ familles $\square$ familles

Source : NRC 2015, 4964 cas, 31/12/2014.

La principale cause de tensions entre réfugiés et Jordaniens est I'accès au logement. Elle est liée à l'augmentation des loyers d'une part et aux expulsions de citoyens jordaniens pauvres à la merci de propriétaires cherchant à rentabiliser leurs biens d'autre part ${ }^{29}$. Tout un habitat dégradé s'est développé, en particulier dans la campagne de Mafraq, afin de loger à un prix élevé des familles syriennes. En quête de logements moins chers ou de meilleures opportunités de travail, les réfugiés sont conduits à changer plusieurs fois de domicile. Ils sont ainsi nombreux dans les quartiers est et sud-est d'Amman, dans la ville industrielle de Zarqa, dans les quartiers pauvres d'Irbid. Fin 2014, un quart des réfugiés syriens en Jordanie n'avait pas de contrat de location, versant d'avance le loyer chaque mois au propriétaire. La moitié de la population des réfugiés n'avait que des contrats de six mois (UNHCR, 2014).

Afin de contribuer à apaiser les tensions liées au logement, le Norwegian Refugee Council a conduit de juillet 2013 à février 2015 un programme qui a consisté à rénover plus de 1000 logements dans le gouvernorat d'Irbid. Les propriétaires jordaniens qui possédaient un logement neuf inachevé, ou ceux qui souhaitaient construire de nouveaux appartements, recevaient en moyenne 3900 JD. En échange de quoi, ils s'engageaient par contrat à héberger gratuitement une famille de réfugiés syriens pendant une période de douze à dix-huit mois selon les contrats. Ce projet a eu un impact très positif tant sur le secteur du logement que sur les relations entre Jordaniens et Syriens (NRC, 2015). Mais il a été suspendu en février 2015 pour des raisons sécuritaires, ne souhaitant pas donner l'impression que les réfugiés étaient appelés à s'installer de manière permanente en Jordanie.

29 Selon une étude conduite en 2014 par REACH, $83 \%$ des Jordaniens et $77 \%$ des Syriens désignent le logement comme première cause de tension (REACH, 2014b). 


\section{Le danger de la réduction de l'aide alimentaire}

La réduction de l'aide distribuée par le Programme d'Aide Alimentaire (PAM) a des conséquences importantes. En 2013, le PAM offrait 24 JD (34 US\$) par personne et par mois hors des camps et 20 JD (28 US\$) par personne et par mois à l'intérieur des camps, sans aucun critère de revenu. Les coupons de nourriture sont échangeables contre des denrées dans quatre-vingt-six centres commerciaux, épiceries et supermarchés de petits villages, faisant de ce fait profiter de nombreux commerçants jordaniens de ce système. Ainsi, vendre des coupons est devenu la deuxième source de revenus des réfugiés. En janvier 2014, le PAM décida d'introduire des critères dits de vulnérabilité (statut matrimonial, âge, handicap, éducation) qui lui ont permis d'exclure 100000 réfugiés de ses registres $^{30}$. Le plus contesté des critères a été d'exclure les réfugiés diplômés du supérieur, aux compétences pourtant inadaptées aux conditions du marché de l'emploi précaire.

En juin 2015, 95000 réfugiés des camps recevaient 20 JD par personne et par mois, 190000 réfugiés extrêmement vulnérables et sans aucune ressource recevaient également $20 \mathrm{JD}$ par personne et par mois, tandis que 240000 réfugiés modérément vulnérables recevaient $10 \mathrm{JD}$ par personne et par mois. En août 2015, ces sommes sont tombées de 20 à 15 JD, pour les plus vulnérables, et à $10 \mathrm{JD}$ pour les modérément vulnérables. Bien que le PAM s'en défende, le gouvernement veille à ce que les montants ne s'effondrent pas pour les réfugiés des camps, cela tant pour prévenir les tensions que pour convaincre les plus démunis de retourner dans les camps (WFP, 2015). En conséquence, un problème sécuritaire majeur pourrait naître si le PAM stoppait en novembre 2015 la distribution d'argent aux résidents des camps, ainsi qu'il a menacé de le faire durant l'été.

\section{L'aide financière aux plus vulnérables}

Dès 2013, le HCR prend en charge financièrement les réfugiés les plus vulnérables, soit les femmes seules, les personnes âgées isolées, les enfants seuls. En 2014, 23200 réfugiés ont reçu un montant mensuel de 50 à 120 JD (selon le nombre de personnes à charge) qu'ils retirent avec des cartes bancaires à des appareils à reconnaissance de l'iris (basmat al ayn). $43 \%$ sont des mères de famille seules et $17 \%$ des personnes âgées dites à risque (UNHCR, 2014).

Les organisations caritatives islamiques, I'Islamic Center Charity Society des Frères musulmans, Kitab wa Sunna et Takaful (Solidarity Association Charity) ont distribué 106,1 millions de dollars d'assistance humanitaire aux réfugiés syriens en Jordanie, sous forme d'aides ponctuelles en liquide (100 à 150 JD par famille), en nourriture, soins et biens de consommation divers (couvertures, habits, bouteilles de gaz). Elles sont d'autant plus actives qu'elles reçoivent un soutien considérable des pays du Golfe, déclaré à 139,8 millions de dollars en 2013 (UNCHR, 2015a). Pour avoir un ordre de comparaison, I'aide totale du HCR

30 Parce que les diverses agences des Nations unies et les grandes ONG utilisaient des critères de vulnérabilité différents, le HCR a lancé en juillet 2014 un projet d'établissement d'une grille commune d'estimation de la vulnérabilité (le VAF, Vulnerability Assessment Framework), dont le rapport est paru en mai 2015 (Washington, Brown et Santacroce, 2015). 
à la Jordanie en 2013 était de 736,6 millions, tandis que le budget du ministère du Développement social est de 125 millions de dinars en $2015^{31}$. Ces organisations caritatives sont enregistrées auprès du ministère du Développement social et coordonnent leur aide via des bases de données électroniques et des cartes de service communes afin de s'assurer que l'aide n'est pas dupliquée. Créée en 1992, Kitab wa Sunna déclare avoir distribué pour 50 millions de dollars d'aide aux réfugiés syriens en 2013. Sa base de données inclut 350000 réfugiés, tous enregistrés au HCR, soit 45817 familles. Régulièrement, cette organisation offre des formations islamiques aux familles, en leur offrant 50 JD la semaine pour $\mathrm{s}^{\prime}$ assurer de leur assiduité ${ }^{32}$.

L'Islamic Center Charity Society a été créé en 1963 en Palestine, en tant que bras caritatif de l'association des Frères musulmans. Elle gère cinquante écoles, deux hôpitaux et vingt dispensaires dans le pays. Sur les deux années 2012 et 2013, elle a aidé 72557 familles syriennes, pour un montant de 37,3 millions de dollars. L'aide n'est mensuelle que pour les veuves (les épouses de " martyrs "). Takaful (" la prise en charge ") est une petite ONG islamique créée en 2010 à Ramtha. Elle distribue essentiellement de la nourriture à 10000 familles syriennes et de l'aide financière à 5000 familles. Elle possède une clinique dont les frais de consultation ne sont que d'1 dinar pour un généraliste et de 2 dinars pour un médecin spécialiste. 200 à 300 réfugiés s'y rendent chaque jour.

\section{La fin de l'aide médicale gratuite pour les réfugiés hors des camps}

De 2011 à 2014, les réfugiés syriens avaient la possibilité de se faire soigner gratuitement dans les hôpitaux jordaniens publics. Mais devant l'augmentation de $10 \%$ des patients et des frais afférents, la politique a été revue. Le 20 novembre 2014, le Cabinet du ministre de la Santé a annoncé la fin de la gratuité des soins dans les hôpitaux et cliniques jordaniens pour les réfugiés syriens, tout en leur donnant un statut particulier, différent de celui des patients étrangers. Désormais, les Syriens doivent payer le tarif des Jordaniens non assurés. Mais les enfants de moins de cinq ans peuvent continuer de bénéficier de la vaccination gratuite. La mesure est rude pour les réfugiés, particulièrement les cancéreux et tous ceux qui suivaient des traitements longs et coûteux qui constituent $12,6 \%$ des réfugiés ${ }^{33}$. C'est une façon d'inciter les plus malades à rejoindre les camps selon les humanitaires interrogés. Hors des camps, les réfugiés peuvent encore bénéficier des deux hôpitaux de la société caritative du centre islamique, qui dépend des Frères musulmans, ainsi que des cliniques ouvertes par les ONG internationales et arabes (UNHCR, 2015).

\footnotetext{
31 Cf. URL : http://petra.gov.jo/Public_News/Nws_NewsDetails.aspx?Site_Id=1\&lang=2 $\&$ NewsID=217834\&Cat|D=-1 et http://www.petra.gov.jo/Public_News/Nws_NewsDetails. aspx?lang=2\&site_id=1\&News $\mid \mathrm{D}=218503 \&$ Cat $\mid \mathrm{D}=13$

32 Elles appliquent la charia concernant les orphelins qui ne sont assistés que jusqu'à leurs treize ans révolus. À quatorze ans, les garçons doivent travailler et les filles attendre d'avoir seize ans pour se marier.

33 UNHCR (2015) External Statistical Report on Active Registered Syrians, 15 September 2015.
} 


\section{Les problèmes de scolarisation des enfants syriens}

Après le logement, et avant que le PAM ne réduise l'aide alimentaire, la deuxième priorité des réfugiés est la scolarisation des enfants. En Jordanie, les enfants syriens dont les parents ont été enregistrés par le ministère de I'Intérieur sont scolarisés. Le dilemme devient très sérieux pour des réfugiés qui hésitent à déménager une fois que les enfants sont scolarisés. En effet, selon la loi jordanienne de l'éducation, un enfant arrivé en cours d'année ne peut pas valider son année scolaire. Fin novembre 2013, 86966 enfants syriens fréquentaient les écoles jordaniennes (MOPIC et HCSP, 2013). En conséquence, $41 \%$ des écoles sont surchargées avec près de cinquante enfants par classe au lieu de vingt-cinq/trente enfants, alors qu'elles n'étaient que $36 \%$ en 2011. Dans quatre-vingt-dix-huit écoles, les cours sont dédoublés en classes du matin pour les Jordaniens (de $7 \mathrm{~h} 00$ à $12 \mathrm{~h} 00$ ) et en classes de l'après-midi pour les Syriens (de $12 \mathrm{~h} 00$ à 17h00). Les sessions de l'après-midi sont réservées aux Syriens, par crainte qu'ils " ne fassent chuter " le niveau des enseignements, alors qu'ils ont tous été déscolarisés pendant plusieurs mois. On a pu observer qu'une partie des enseignants recrutés dans l'urgence n'a pas le niveau requis (INEE, 2015).

Mais les Jordaniens se plaignent surtout des mauvaises conditions d'enseignement pour leurs enfants et d'infrastructures sanitaires qui se dégradent. Les enfants syriens sont eux bien souvent victimes de harcèlement sur le chemin de l'école, certains enseignants allant même jusqu'à leur interdire l'utilisation des toilettes. Devant cette situation, les tensions pèsent sur la scolarité et sur les relations entre les enfants. L'appauvrissement des réfugiés, surtout à partir de 2015, a pour conséquence la baisse de la scolarisation des enfants syriens. Alors qu'ils étaient 60 \% à être scolarisés en 2014, ils ne sont plus que la moitié en juillet 2015. Le nombre d'enfants contraints de travailler augmente, ce qui les expose à des risques $d^{\prime}$ abus de diverses natures.

\section{Le travail informel des réfugiés en Jordanie}

Le gouvernement a interdit aux réfugiés syriens de travailler, répondant aux inquiétudes des Jordaniens pensant qu'ils allaient entrer en concurrence avec eux ${ }^{34}$. Moins de $1 \%$ des réfugiés a un membre de sa famille qui a obtenu un contrat de travail (UNHCR, 2015), souvent dans l'agriculture, car il ne coûte que 250 JD. Pourtant, le Bureau International du Travail estimait le nombre de travailleurs syriens à 160000 en 2013 (Ajluni et Kawar, 2014). Selon la base de données du Norwegian Refugee Council (NRC) en décembre 2014, 60,1 \% des réfugiés paient leurs loyers grâce à leur travail informel, 11,8 \% grâce à des donations d'ONG et de particuliers, 9,6\% par leurs économies, 9,5\% par l'assistance financière offerte aux plus démunis par le HCR et $9 \%$ sont insolvables (Figure 5) (NRC, 2015).

\footnotetext{
34 Le département des statistiques a publié en décembre 2014 le Household Income and Expenditure Survey pour les gouvernorats de Mafraq et d'Irbid. On y lit l'exaspération grandissante des Jordaniens des gouvernorats d'Irbid et Mafraq. 92 \% des 4600 personnes interrogées ont déclaré que l'arrivée des Syriens était la raison de l'augmentation des prix et $55 \%$ que leurs revenus avaient diminué du fait des réfugiés. Cf. URL : http://www.hostcommunities-jo.org/photo-gallery/publications/reports/
} 
Figure 5 : Source de paiement des loyers en milieu urbain par les réfugiés syriens en Jordanie

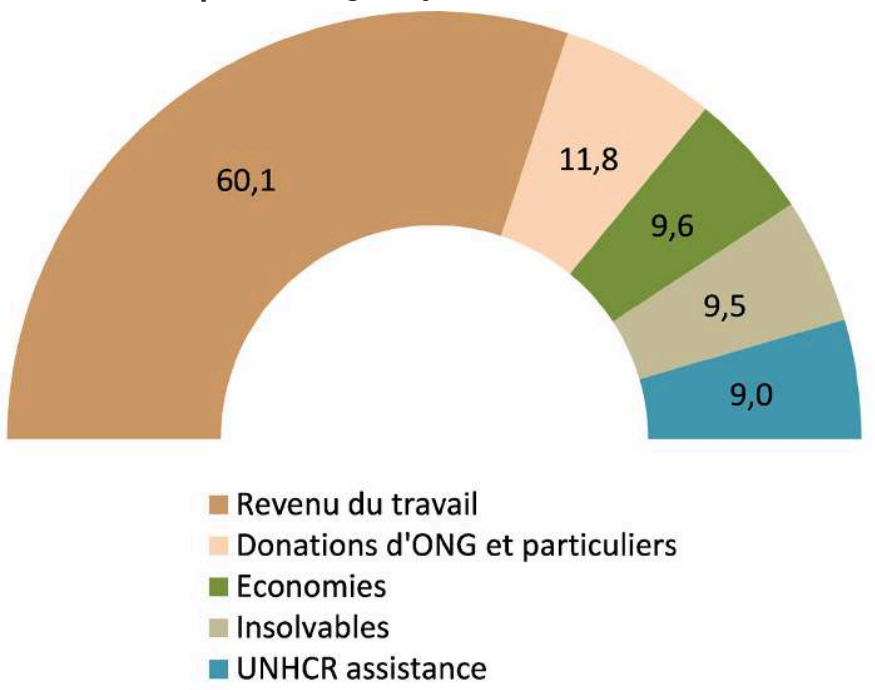

Source : NRC, 2015.

Les réfugiés syriens sont entrés en concurrence avec les travailleurs égyptiens, estimés à 500000 dans le pays, dont 176000 sans permis de travail. Mais si le travail des Jordaniens s'est réduit dans le secteur de la construction et que le chômage a augmenté à Amman, Mafraq et Irbid selon I'enquête conduite par le BIT en partenariat avec le centre de recherche norvégien Fafo et le département des statistiques, les Syriens ont eu un impact stimulant sur le marché du travail (Stave Svein et Hillesund, 2015 ; Carrion, 2015). En acceptant des salaires très bas et en étant polyvalents, les réfugiés syriens ont redynamisé les petites et moyennes entreprises jordaniennes. Certains Syriens ont même créé des entreprises, dont les employés étaient en majorité syriens, mais qui ont bénéficié aux Jordaniens (nombreux restaurants, ateliers de couture, de menuiserie, salons de coiffure). Enfin, toute l'économie de l'aide humanitaire bénéficie également aux Jordaniens, via les nombreux commerces qui distribuent l'aide alimentaire. Le BIT tente de négocier avec le gouvernement I'ouverture aux réfugiés des secteurs de la construction et de l'agriculture. Si quelques maires proposent de faire travailler les réfugiés comme cantonniers (Chatham House, 2015), des autorités jordaniennes n'hésitent pas à proposer de remplacer progressivement les travailleurs égyptiens, qui sont en majorité analphabètes, ainsi que les travailleurs asiatiques embauchés dans les zones industrielles qualifiées par des réfugiés syriens. Le gouvernement pense développer des zones économiques spéciales dans lesquelles les réfugiés seraient employés (Carrion, 2015).

\section{Conclusion}

Avec plus d'1 million de réfugiés syriens, sur une population totale de 6,5 millions, la Jordanie est confrontée à de nouveaux défis. L'aide internationale 
humanitaire est en réduction, alors que les efforts du gouvernement se portent sur le secteur de la défense, dans le cadre de la participation de la Jordanie aux forces de la coalition. Si la Jordanie dit maintenir ses frontières ouvertes à l'accueil de réfugiés, dans les faits, ces derniers sont soumis à des contrôles croissants. Dans le contexte sécuritaire grandissant, le principe de non-refoulement n'est plus respecté et de nombreux Syriens sont renvoyés en Syrie, dès qu'ils sont suspectés de soutenir l'État islamique ou d'être des combattants. Le pays a déployé tout un dispositif de contrôle des réfugiés. Des enregistrements biométriques par l'empreinte de l'iris sont effectués, qui permettent au HCR de distribuer l'aide aux plus démunis, et au ministère de I'Intérieur d'émettre des cartes de service pour les réfugiés. Depuis 2014, une politique de retour des réfugiés vers les camps est conduite (encampement policy), afin de répondre aux demandes de la population exaspérée par l'augmentation du chômage et la dégradation du service scolaire public. Plus de $85 \%$ des réfugiés vivent sous le seuil de pauvreté, et ont du mal à payer les loyers élevés demandés dans les villes du Nord du pays. En conséquence, le nombre d'enfants scolarisés se réduit à $50 \%$. Un mouvement de retour vers la Syrie en guerre s'est amorcé à I'automne 2015 et concerne 300 personnes par jour. Certains tentent de regagner la Turquie, puis l'Europe.

La Jordanie est confrontée à un autre défi de taille : maintenir une politique de développement et de croissance, alors que ses voies routières de communication vers l'Europe via la Syrie et la Turquie sont fermées, et offrir de nouvelles opportunités d'emploi et de logement aux Jordaniens mis en concurrence avec les Syriens dans le Nord du pays, tout en contenant la montée des extrémismes. Alors que les autorités jordaniennes prévoient que la crise durera encore dix ans, le gouvernement se prépare à des stratégies d'intégration des réfugiés syriens dans le secteur de l'emploi formel. Il s'agit de pouvoir bénéficier des talents et de la créativité de migrants. Le gouvernement cherche aussi à se prémunir contre la radicalisation possible de cette population pauvre et jeune qui, si elle ne subit plus l'endoctrinement du régime syrien, est en quête de repères.

\section{Références bibliographiques}

Ababsa Myriam (2013a) Atlas of Jordan. History, Territories and Society. Atlas al Urdunn. Al-tarikh, al-ardh, al-mujtama', Beyrouth, IFPO, 485 p., [online]. URL: http://books.openedition.org/ifpo/4560

Ababsa Myriam (2013b) Deux pas en avant, un pas en arrière. Comment réformer la Jordanie?, Moyen-Orient, 19, pp. 68-73.

Achilli Luigi (2015) Syrian Refugees in Jordan: A Reality Check, European University Institute, Migration Policy Centre, $12 \mathrm{p}$.

Ajluni Salem and Kawar Mary (2014) The Impact of the Syrian Refugee Crisis on the Labour Market in Jordan: Preliminary Analysis, [online]. URL: http://www. ilo.org/wcmsp5/groups/public/---arabstates/---ro-beirut/documents/publication/ wcms_242021.pdf

Akram Susan et al. (2014) Protecting Syrian Refugees: Laws, Policies, and Global Responsibility Sharing, Boston, Boston University, 139 p., [online]. URL: http://www.bu.edu/law/central/jd/programs/clinics/international-human-rights/ documents/FINALFulIReport.pdf 
Al Husseini Jalal (2013) La Jordanie face à la crise syrienne, in François Burgat et Bruno Paoli, Pas de Printemps pour la Syrie, Paris, La Découverte, pp. 282-288.

Al Husseini Jalal (2011) The Evolution of the Palestinian Refugee Camps in Jordan: between Logics of Exclusion and Integration, in Myriam Ababsa et Rami Daher, Villes, pratiques urbaines et construction nationale en Jordanie. Cities, Urban Practices and Nation-Building in Jordan, Beyrouth, Presses de I'Ifpo, pp. 181-204, [online]. URL: http://ifpo.revues.org/1742

Al Kilani Saleh (2014) A duty and a burden for Jordan, Forced Migration Review: The Syria crisis, displacement and protection, pp. 30-31, [online]. URL: http:// www.fmreview.org/en/syria/alkilani.pdf

Al Wazani Khalid (2014) The Socio-Economic Implications of Syrian Refugees on Jordan, Amman, Konrad Adenauer Stiftung.

Amnesty International (2013) Growing Restrictions, Tough Conditions: The Plight of Those Fleeing Syria To Jordan, October 2013, [online]. URL: http://www. amnestyusa.org/research/reports/growing-restrictions-tough-conditions-theplight-of-those-fleeing-syria-to-jordan

Caillet Romain (2011) Note sur I'espace public salafi en Jordanie, in Myriam Ababsa et Rami Daher, Villes, pratiques urbaines et construction nationale en Jordanie. Cities, Urban Practices and Nation-Building in Jordan, Beyrouth, Presses de I'Ifpo, [en ligne]. URL : http://ifpo.revues.org/1675

Care (2014) Lives Unseen: Urban Syrian Refugees and Jordanian Host Communities Three Years into the Syria Crisis, [online]. URL: http://reliefweb.int/ report/jordan/lives-unseen-urban-syrian-refugees-and-jordanian-host-communities-three-years-syria

Care (2013) Syrian Refugees in Urban Jordan Report, [online]. URL: http://www. care.org/syrian-refugees-urban-jordan

Carrion Doris (2015) Syrian Refugees in Jordan. Confronting Difficult Truths, Chatham House, the Royal Institute of International Affairs, Middle East and North Africa Program, Research Paper, 15 p.

Chatelard Geraldine (2009) What visibility conceals. Re-embedding Refugee Migration from Iraq, in Dawn Chatty and Bill Finlayson Dirs., Dispossession and Displacement: Forced-Migration in the Middle East and North Africa, Oxford, Oxford University Press, British Academy Occasional Papers 14, [online]. URL: https://halshs.archives-ouvertes.fr/halshs-00370980

Chatham House (2015) The Role of Local Government in Addressing the Impact of Syrian Refugees: Jordan Case Study, Workshop Summary, 2-3 June 2015, [online]. URL: http://www.chathamhouse.org/sites/files/chathamhouse/field/ field_document/Jordan\%20workshop\%20summary\%20FINAL.pdf

Curtis Ryan (2014) Jordanian Foreign Policy and the Arab Spring, in Middle East Policy, XXI (1), [online]. URL: http://www.mepc.org/journal/middle-east-policyarchives/jordanian-foreign-policy-and-arab-spring?print

De Bel-Air Françoise (2013) Composition of the population, in Myriam Ababsa, Atlas of Jordan. History, Territories and Society. Atlas al Urdunn. Al-tarikh, al-ardh, al-mujtama', Beyrouth, IFPO, pp. 246-252.

Dhingra Reva (2014) Syrian Refugees and the Jordanian Economy, Muftah, [online]. URL: http://muftah.org/syrian-refugees-jordanian-economy/ 
Fafo (2007) Iraqis in Jordan. Their Number and Characteristics, 116 p., [online]. URL: http://www.google.com/url?sa=t\&rct=j\&q=\&esrc=s\&source=web\&cd=1\& cad=rja\&uact=8\&ved=0ahUKEwjJ8dr5_dPLAhXLESwKHba7CucOFggdMAA\&ur $\mathrm{I}=\mathrm{http} \% 3 \mathrm{~A} \% 2 \mathrm{~F} \% 2 \mathrm{Fwww}$.unhcr.org\%2F47626a232.pdf\&usg=AFQjCNGnOCui5JEW3MOKfvdiGPLev1hHQ\&sig2=hlsOmmRFwMxD-c3-bpvaoO

Hassan Hanine (2014) Cyber City in Jordan: the Epitome of Palestine Refugees' Despair and Legal Limbo, BADIL, 56, [online]. URL: http://www.badil.org/en/ publication/periodicals/al-majdal/item/2070-article-7.html

Hasselbarth Sarah (2014) Islamic Charities in the Syrian Context in Jordan and Lebanon, Beirut, Friedrich Ebert Stiftung, $44 \mathrm{p}$.

Human Rights Watch (2015) Jordan: Syrians Blocked, Stranded in Desert, [online]. URL: https://www.hrw.org/news/2015/06/03/jordan-syrians-blockedstranded-desert

Human Rights Watch (2014) Not Welcome. Jordan's Treatment of Palestinians Escaping Syria, $44 \mathrm{p}$.

Hyndman Jennifer (2000) Managing Displacement. Refugees and the Politics of Humanitarianism, Minneapolis, University of Minnesota Press, 253 p.

INEE (The Inter-Agency Network for Education in Emergencies) and ESWG (Jordan Education Sector Working Group) (2015) Jordan Minimum Standards for Education in Emergencies, 118 p.

IRIN (2013) Despite new police presence, security concerns persist a Syrian refugee camp, [online]. URL: http://www.irinnews.org/report/97778/despite-newpolice-presence-security-concerns-persist-at-syrian-refugee-camp

JRC (Jordan Red Crescent) (2012) Syrian Refugees living in the Community in Jordan. Assessment Report, $55 \mathrm{p}$.

Lamloum Imed (2015) Réfugiés syriens. En Jordanie l'approche sécuritaire prime sur I'humanitaire, L'Orient-Le Jour, [en ligne]. URL : http://www.lorientlejour. com/article/943962/refugies-syriens-en-jordanie-lapproche-securitaire-primesur-lhumanitaire.html

MOPIC and HCSP (Host Community Support Platform) (2013) Needs Assessment Review of the Impact of the Syrian Crisis on Jordan, $14 \mathrm{p}$.

NRC (Norwegian Refugee Council) (2015) In Search of a Home. Access to adequate housing in Jordan, 36 p. [online]. URL: https://www.nrc.no/arch/_ img/9200922.pdf

Oddone Elisa and Reznick Alisa (2015) Refugee Camps Empty as Syrians Head for Europe, Time, 07/10/2015, [online]. URL: http://time.com/4063674/zaataricamps-empty-syria/

Olwan Mohammed (2007) The Legal Framework of Forced Migration and Refugee Movements in Jordan, Paper Prepared for The Forced Migration \& Refugee Studies Program, 23-25/10/2007, Cairo, The American University in Cairo.

Olwan Mohammed and Shiyab Ahmed (2012) Forced Migration of Syrians to Jordan: An Exploratory Study, MPC Research Report 2012/06, 31 p., [online]. URL: http://www.migrationpolicycentre.eu/docs/MPC\%202012\%20EN\%2006.pdf

Puig Nicolas et Doraï Kamel (Éds.) (2012) L'urbanité des marges. Migrants, réfugiés et relégués dans les villes du Proche-Orient, Paris, Téraèdre, Ifpo, 335 p. 
REACH (2014a) Evaluating the Effect of the Syrian Refugee Crisis on Stability and Resilience in Jordanian Host Communities, 44 p. [online]. URL: http:// www.reach-initiative.org/wp-content/uploads/2014/02/jeffrey.frankens10022014-093154-REACH-FCO_Syrian-Refugees-in-Host-Communities_ Preliminary-Impact-Assessment.pdf

REACH (2014b) Housing and Tensions in Jordanian Communities Hosting Syrian Refugees. Thematic Assessment Report, 15 p.

REACH (2014c) Syrian Refugees Staying, in Informal Tented Settlements in Jordan, Multi-Sector Assessment Report, $44 \mathrm{p}$.

Refugee Council USA (2015) At the Breaking Point: Refugees in Jordan and Egypt, 25 p., [online]. URL: http://www.rcusa.org/uploads/pdfs/At $\% 20$ the $\% 20$ Breaking \%20Point \%20-\%20RCUSA\%20Report\%20on\%20Refugees\%20in\%20 Jordan\%20and\%20Egypt.pdf

Seeley Nicolas (2010) The Politics of Aid to Iraqi Refugees in Jordan, MERIP, Report 256, [online]. URL: http://www.merip.org/mer/mer256/politics-aid-iraqirefugees-jordan

Sowell Kirk H. (2015) Jordanian Salafism and the Jihad in Syria, Hudson Institute, [online]. URL: http://www.hudson.org/research/11131-jordanian-salafism-andthe-jihad-in-syria

Stave Svein Erik and Hillesund Solveig (2015) Impact of Syrian refugees on the Jordanian labour market, ILO, Fafo, Dos, 160 p.

UNHCR (2016) Syria Regional Refugee Response: Inter-agency Information Sharing Portal, [online]. URL: http://data.unhcr.org/syrianrefugees/country. php?id=107.

UNHCR (2015a) Gulf Donors and NGOs Assistance to Syrian refugees in Jordan, 62 p.

UNHCR (2015b) Living in the Shadow, 80 p., [online]. URL: http://www.unhcr. org/54b685079.pdf

UNHCR (2014) A Year in Review. UNHCR Jordan, 28 p.

UNHCR-ISWG (2015) Jordan Inter-Sector Working Group Update, 6 p.

UNHCR, UNICEF and WFP (2014) Joint Assessment Review of the Syrian Refugee Response in Jordan, [online]. URL: http://reliefweb.int/report/jordan/joint-assessment-review-syrian-refugee-response-jordan

UPP (Un Ponte Per) (2012) Comprehensive Assessment on Syrian Refugees residing in the community in northern Jordan, 17 p., [online]. URL: http:// reliefweb.int/report/jordan/comprehensive-assessment-syrian-refugeesresiding-community-northern-jordan

UN Women (2013) Inter-agency Assessment: Gender-Based Violence and Child Protection among Syrian Refugees in Jordan, with a Focus on Early Marriage, 92 p. [online]. URL: http://resourcecentre.savethechildren.se/sites/default/files/ documents/un_women_gbv_and_cp_assessment_report_-_syrian_refugees_in_ jordan1.pdf

Washington Kate, Brown Harry and Santacroce Marco (2015) Jordan Refugee Response. Vulnerability Assessment Framework. Baseline Survey, UNHCR, 106 p.

WFP (World Food Program) (2015) Frequently Asked Questions. Topic: August changes for Syrian refugees living outside of camps. 


\section{Myriam Ababsa \\ De la crise humanitaire à la crise sécuritaire. Les dispositifs de contrôle des réfugiés syriens en Jordanie (2011-2015)}

Avec près de $20 \%$ de sa population formée de réfugiés syriens, la Jordanie est confrontée à de grands défis tant économiques que policiers. La pression que font peser les réfugiés sur les infrastructures scolaires et les services médicaux est telle que la crise humanitaire se transforme en crise politique sur le plan intérieur et en crise sécuritaire. Sous la pression de l'opinion publique et parce qu'elle a décidé de s'engager militairement dans le combat contre l'El, la Jordanie a durci sa politique envers les réfugiés syriens en 2014. L'interdiction de travail est maintenue, des contrôles sont conduits et les conditions de résidence sont de plus en plus restrictives. Les réfugiés sont triés et certains refoulés, ce qui est contraire aux résolutions internationales. Le gouvernement tente de repeupler les trois camps de réfugiés en y dirigeant les Syriens sans papier. L'objectif de cet article est de présenter l'évolution de la politique de gestion jordanienne des réfugiés syriens depuis quatre ans, en mettant l'accent sur l'évolution des dispositifs de contrôle et des cadres légaux, sous la pression du contexte géopolitique de guerre contre les djihadistes.

\section{$\cdots$ From a Humanitarian Crisis to a Security Crisis. Control Measures for Syrian Refugees in Jordan (2011-2015)}

With refugees constituting nearly $20 \%$ of its population, Jordan is confronted with serious economic and political challenges. So great is the strain the refugee community is putting on the education system and the health services in Jordan that the humanitarian crisis is now becoming a political issue on the domestic level and a security crisis. In response to increased public pressure and the decision to partake in airstrikes against the Islamic State, Jordan toughened its policy against Syrian refugees in 2014. Under this stricter policy, refugees continue to be forbidden to work and the conditions for attaining residence are becoming increasingly more restrictive. The aim of this article is to present the evolution of the management policy of the Jordanian government for refugees over the past four years. The article will analyse first and foremost the development of control measures and legal frameworks within the pressure of the geopolitical context of the war against jihadists.

\section{De la crisis humanitaria a la crisis de seguridad. Los dispositivos de vigilancia de los refugiados sirios en Jordania (2011-2015)}

Con cerca de un $20 \%$ de su población constituida por refugiados sirios, Jordania hace frente a desafíos tanto económicos como de carácter policial. La presión que constituyen los refugiados sobre las infraestructuras escolares y sobre los servicios médicos es tal que la crisis humanitaria se está transformando en crisis política interna y en crisis en materia de seguridad. Bajo presión de la opinión pública y dado que el país ha decidido comprometerse militarmente en la lucha contra el Estado Islámico, Jordania ha endurecido su política con respecto a los refugiados sirios en 2014. La prohibición de trabajar se mantiene, se realizan controles y las condiciones de residencia son cada vez más restrictivas. Los refugiados son clasificados y algunos reenviados, lo cual es contrario a las resoluciones internacionales. El gobierno intenta repoblar los tres campos de refugiados orientando hacia ellos a los sirios sin papeles. El objetivo de este artículo es presentar la evolución de la política jordana de gestión de los refugiados sirios en los últimos cuatro años, insistiendo en la evolución de los dispositivos de control y en los marcos legales bajo la presión de un contexto geopolítico de guerra contra los yihadistas. 